\title{
OGLE-2013-BLG-0911Lb: A Secondary on the Brown-dwarf Planet Boundary around an M Dwarf
}

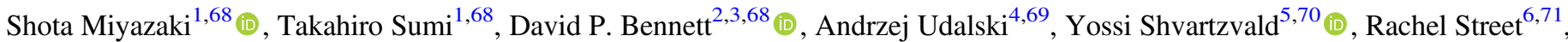
Valerio Bozza ${ }^{7,8,72}$ (D) , Jennifer C. Yee ${ }^{9,73}$ (D), Ian A. Bond ${ }^{10}$, Nicholas Rattenbury ${ }^{11}$ (i), Naoki Koshimoto ${ }^{1}$ (i), Daisuke Suzuki ${ }^{12}$, Akihiko Fukui ${ }^{13,14}$ (iD, and

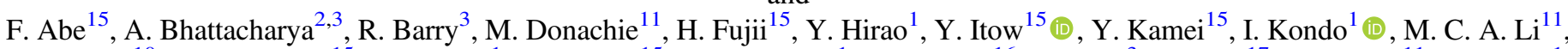
C. H. Ling ${ }^{10}$, Y. Matsubara ${ }^{15}$, T. Matsuo ${ }^{1}$, Y. Muraki ${ }^{15}$, M. Nagakane ${ }^{1}$, K. Ohnishi ${ }^{16}$, C. Ranc ${ }^{3}$, T. Saito ${ }^{17}$, A. Sharan ${ }^{11}$, H. Shibai ${ }^{1}$, H. Suematsu ${ }^{1}$, D. J. Sullivan ${ }^{18}$, P. J. Tristram ${ }^{19}$, T. Yamakawa ${ }^{15}$, A. Yonehara ${ }^{20}$

(MOA collaboration),

J. Skowron ${ }^{4}$ (1), R. Poleski ${ }^{21}$, P. Mróz ${ }^{4,22}$, M. K. Szymański ${ }^{4}$, I. Soszyński ${ }^{4}$, P. Pietrukowicz ${ }^{4}$ (1) , S. KozŁowski ${ }^{4}$, K. Ulaczyk $^{4}$ (1), Ł. Wyrzykowski ${ }^{4}$

(OGLE collaboration), Matan Friedmann ${ }^{23}$, Shai Kaspi ${ }^{23}$, Dan $\mathrm{Maoz}^{23}$

(Wise team),

M. Albrow ${ }^{24}$ (1) , G. Christie ${ }^{25}$, D. L. DePoy ${ }^{26}$, A. Gal-Yam ${ }^{27}$ (1) A. Gould ${ }^{28,29,30}$, C.-U. Lee ${ }^{31,32}$, I. Manulis ${ }^{27}$, J. McCormick ${ }^{33}$,

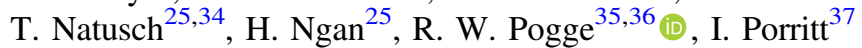

$(\mu \mathrm{FUN})$,

Y. Tsapras ${ }^{38}$ (1) , E. Bachelet ${ }^{6,39}$ (1), M. P. G. Hundertmark ${ }^{38}$, M. Dominik ${ }^{40}$, D. M. Bramich ${ }^{41,42,43}$, A. Cassan ${ }^{44}$,

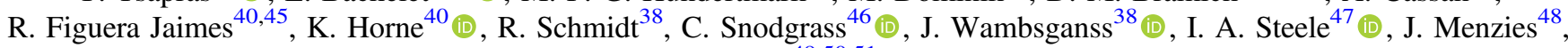
S. Mao ${ }^{49,50,51}$

(RoboNet),

and

U. G. Jø rgensen ${ }^{52}$, M. J. Burgdorf $5^{53}$, S. Ciceri $^{30}$, S. Calchi Novati ${ }^{54}$, G. D’Ago ${ }^{7,8,55}$ ๑ , D. F. Evans ${ }^{56}$, T. C. Hinse ${ }^{57}$ (1),

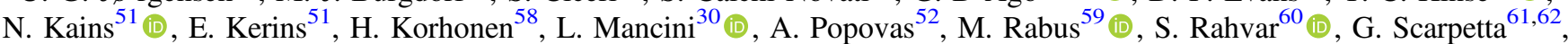
J. Skottfelt ${ }^{52,63}$, J. Southworth ${ }^{56}$, G. D’Ago ${ }^{64}$, N. Peixinho ${ }^{65,66}$ (1) , and P. Verma ${ }^{67}$

(MiNDSTEp)

${ }^{1}$ Department of Earth and Space Science, Graduate School of Science, Osaka University, 1-1 Machikaneyama, Toyonaka, Osaka 560-0043, Japan ${ }^{2}$ Department of Physics, University of Notre Dame, Notre Dame, IN 46556, USA

${ }^{3}$ Laboratory for Exoplanets and Stellar Astrophysics, NASA/Goddard Space Flight Center, Greenbelt, MD 20771, USA

${ }^{4}$ Warsaw University Observatory, Al. Ujazdowskie 4, 00-478 Warszawa, Poland

${ }^{5}$ IPAC, Mail Code 100-22, Caltech, 1200 East California Boulevard, Pasadena, CA 91125, USA

${ }^{6}$ Las Cumbres Observatory Global Telescope Network, 6740 Cortona Drive, Suite 102, Goleta, CA 93117, USA

${ }^{7}$ Dipartimento di Fisica "E.R. Caianiello,"Universitá di Salerno, Via Giovanni Paolo II 132, I-84084, Fisciano, Italy ${ }^{8}$ Istituto Nazionale di Fisica Nucleare, Sezione di Napoli, Napoli, Italy

${ }^{9}$ Center for Astrophysics|Harvard \& Smithsonian, 60 Garden Street, Cambridge, MA 02138, USA

${ }^{10}$ Institute of Information and Mathematical Sciences, Massey University, Private Bag 102-904, North Shore Mail Centre, Auckland, New Zealand ${ }^{11}$ Department of Physics, University of Auckland, Private Bag 92019, Auckland, New Zealand

${ }_{12}^{12}$ Institute of Space and Astronautical Science, Japan Aerospace Exploration Agency, 3-1-1 Yoshinodai, Chuo, Sagamihara, Kanagawa 252-5210, Japan

${ }^{13}$ Department of Earth and Planetary Science, Graduate School of Science, The University of Tokyo, 7-3-1 Hongo, Bunkyo-ku, Tokyo 113-0033, Japan

${ }^{14}$ Instituto de Astrofísica de Canarias, Vía Láctea s/n, E-38205 La Laguna, Tenerife, Spain

${ }^{15}$ Institute for Space-Earth Environmental Research, Nagoya University, Nagoya 464-8601, Japan

${ }^{16}$ Nagano National College of Technology, Nagano 381-8550, Japan

${ }^{17}$ Tokyo Metropolitan College of Industrial Technology, Tokyo 116-8523, Japan

${ }^{18}$ School of Chemical and Physical Sciences, Victoria University, Wellington, New Zealand

${ }^{19}$ University of Canterbury Mt. John Observatory, P.O. Box 56, Lake Tekapo 8770, New Zealand

20 Department of Physics, Faculty of Science, Kyoto Sangyo University, Kyoto 603-8555, Japan

${ }^{21}$ Department of Astronomy, Ohio State University, 140 W. 18th Avenue, Columbus, OH 43210, USA

${ }^{22}$ Division of Physics, Mathematics, and Astronomy, California Institute of Technology, Pasadena, CA 91125, USA

${ }^{23}$ School of Physics and Astronomy and Wise Observatory, Tel-Aviv University, Tel-Aviv 6997801, Israel

${ }^{24}$ Department of Physics and Astronomy, University of Canterbury, Private Bag 4800, Christchurch, New Zealand ${ }^{25}$ Auckland Observatory, Auckland, New Zealand

${ }^{26}$ Department of Physics and Astronomy, Texas A\&M University, College Station, TX 77843-4242, USA

27 Department of Particle Physics and Astrophysics, Weizmann Institute of Science, 76100 Rehovot, Israel

${ }^{28}$ Department of Astronomy, Ohio State University, 140 West 18th Avenue, Columbus, OH 43210, USA

${ }^{29}$ Korea Astronomy and Space Science Institute, Daejon 34055, Republic of Republic of Korea

${ }^{30}$ Max Planck Institute for Astronomy, Königstuhl 17, D-69117 Heidelberg, Germany

${ }^{31}$ Korea Astronomy and Space Science Institute, 776 Daedukdae-ro, Daejeon, Republic of Korea

${ }^{32}$ University of Science and Technology, Korea, (UST), 217 Gajeong-ro Yuseong-gu, Daejeon 34113, Republic of Korea

${ }^{33}$ Farm Cove Observatory, Centre for Backyard Astrophysics, Pakuranga, Auckland, New Zealand

${ }^{34}$ Institute for Radio Astronomy and Space Research (IRASR), AUT University, Auckland, New Zealand

${ }^{35}$ Department of Astronomy, The Ohio State University, 140 W 18th Avenue, Columbus, OH 43210, USA

${ }^{36}$ Center for Cosmology \& AstroParticle Physics, The Ohio State University, 191 West Woodruff Avenue, Columbus, OH 43210, USA 


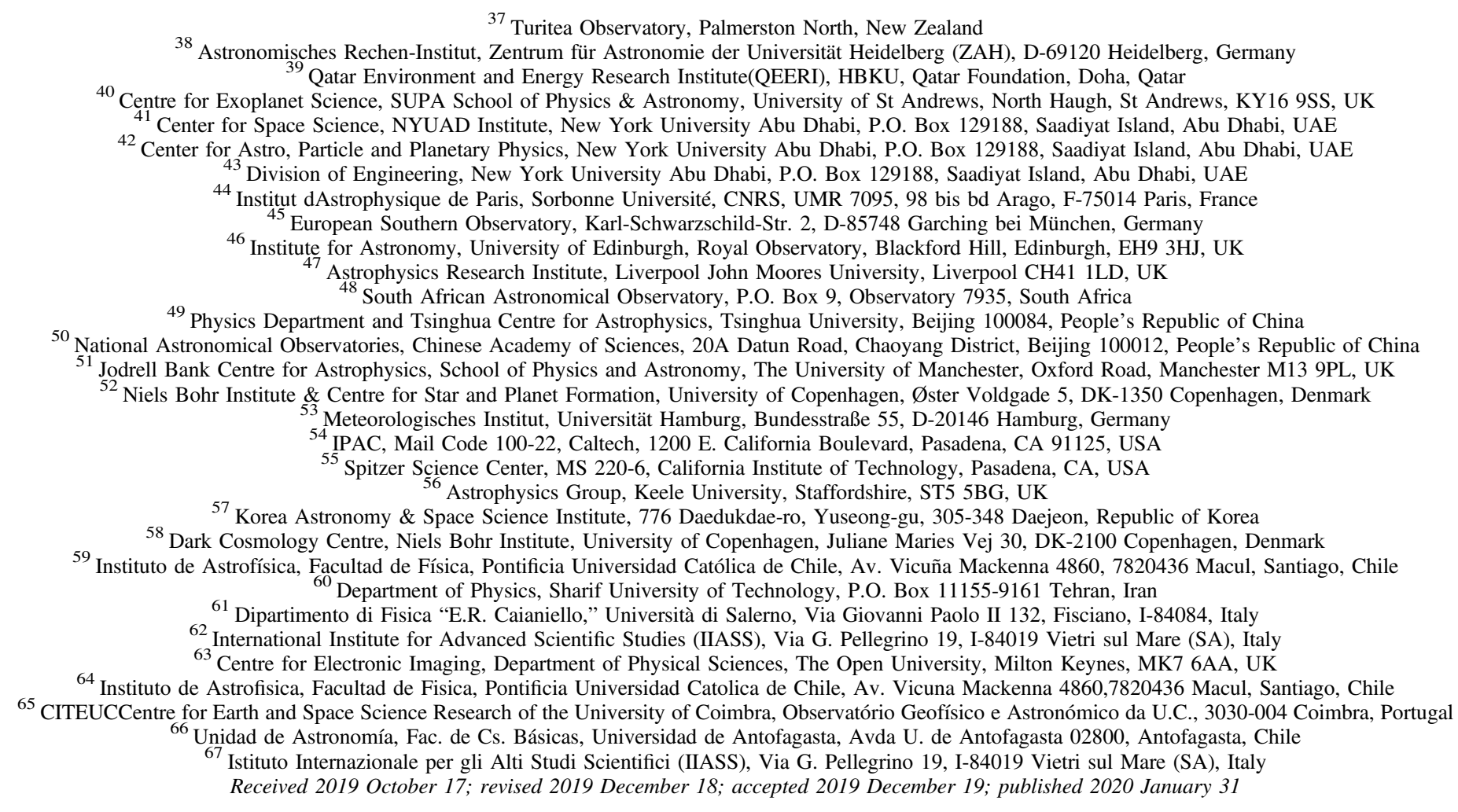

\begin{abstract}
We present the analysis of the binary-lens microlensing event OGLE-2013-BLG-0911. The best-fit solutions indicate the binary mass ratio of $q \simeq 0.03$, which differs from that reported in Shvartzvald et al. The event suffers from the well-known close/wide degeneracy, resulting in two groups of solutions for the projected separation normalized by the Einstein radius of $s \sim 0.15$ or $s \sim 7$. The finite source and the parallax observations allow us to measure the lens physical parameters. The lens system is an $\mathrm{M}$ dwarf orbited by a massive Jupiter companion at very close $\left(M_{\text {host }}=0.30_{-0.06}^{+0.08} M_{\odot}, M_{\text {comp }}=10.1_{-2.2}^{+2.9} M_{\text {Jup }}, a_{\text {exp }}=0.40_{-0.04}^{+0.05}\right.$ au $)$ or wide $\left(M_{\text {host }}=0.28_{-0.08}^{+0.10} M_{\odot}\right.$, $M_{\text {comp }}=9.9_{-3.5}^{+3.8} M_{\text {Jup }}, a_{\text {exp }}=18.0_{-3.2}^{+3.2}$ au) separation. Although the mass ratio is slightly above the planet-brown dwarf (BD) mass-ratio boundary of $q=0.03$, which is generally used, the median physical mass of the companion is slightly below the planet-BD mass boundary of $13 M_{\mathrm{Jup}}$. It is likely that the formation mechanisms for BDs and planets are different and the objects near the boundaries could have been formed by either mechanism. It is important to probe the distribution of such companions with masses of $\sim 13 M_{\text {Jup }}$ in order to statistically constrain the formation theories for both BDs and massive planets. In particular, the microlensing method is able to probe the distribution around low-mass M dwarfs and even BDs, which is challenging for other exoplanet detection methods.
\end{abstract}

Unified Astronomy Thesaurus concepts: Gravitational microlensing (672); Exoplanet astronomy (486); Brown dwarfs (185); Exoplanet systems (484); Exoplanets (498); Extrasolar gas giants (509); Exoplanet detection methods (489); M dwarf stars (982)

\section{Introduction}

Brown dwarfs (BDs) have masses of $13-75 M_{\text {Jup }}$, as they are intermediate between the masses of main-sequence stars and planets (Burrows et al. 1993). Although the existence of BDs was first proposed in Kumar (1962), there had been no observational evidence for BDs until 1995 (Nakajima et al. 1995) owing to their low luminosities and temperatures. To date, more than 10,000 field BDs have been discovered by

\footnotetext{
68 MOA collaboration.

69 OGLE collaboration.

70 Wise team.

71 RoboNet.

72 MiNDSTEp.

$73 \mu \mathrm{FUN}$.
}

several survey groups, which are summarized in Table 1 of Carnero Rosell et al. (2019). Most current theories predict that field BDs are formed in a fashion similar to that of mainsequence stars, through direct gravitational collapse and turbulent fragmentation of molecular clouds (Luhman 2012). These theories are observationally supported. For example, André et al. (2012) found self-gravitating dense clumps of gases and dust with mass $0.015-0.03 M_{\odot}$, which are similar to those of low-mass BDs. On the other hand, the core accretion mechanism (Mordasini et al. 2009; Tanigawa \& Tanaka 2016) and that of gravitational instability (Boss 1997, 2001) are also able to produce companions of BD masses in protoplanetary disks. Radial velocity (RV) surveys have revealed that the frequency of BD companions with orbital radii less than $\sim 3$ au around main-sequence stars is relatively lower than that of 


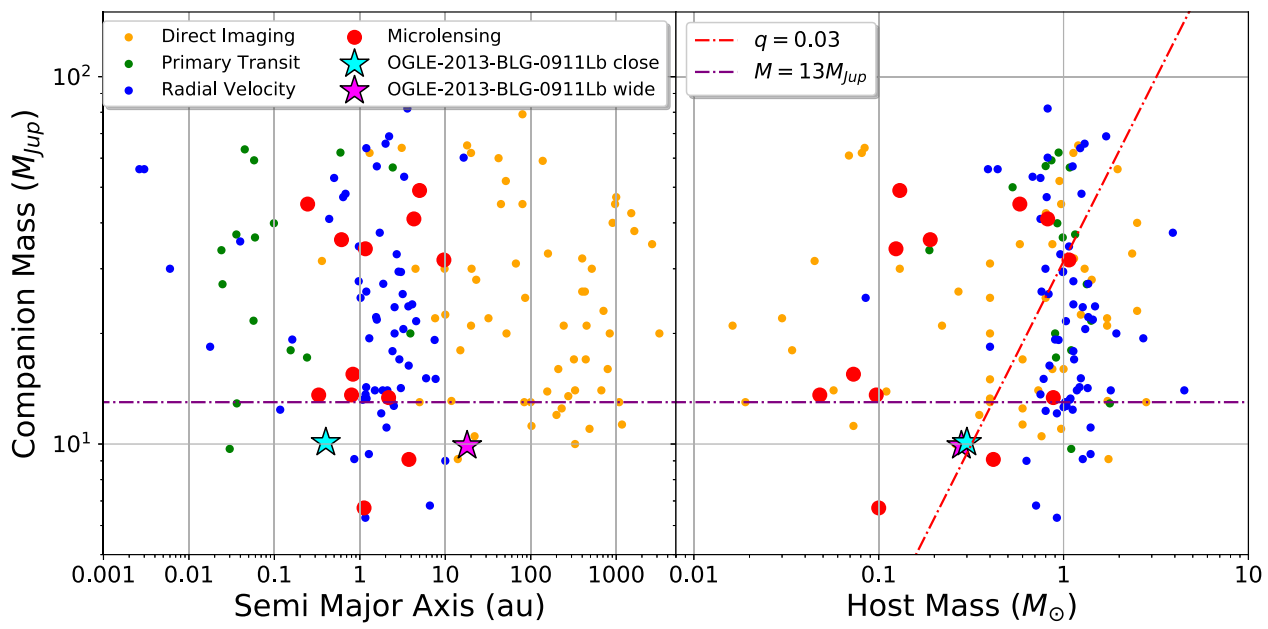

Figure 1. Distributions of discovered $\mathrm{BD} /$ massive-planet companions ( $5 M_{\mathrm{Jup}} \leqslant M \leqslant 75 M_{\mathrm{Jup}}$ ) obtained from http://exoplanet.eu, in which the vertical axis shows the companion masses. The horizontal axes for the left and right panels indicate the semimajor axes and host masses, respectively. The yellow, green, blue, and red points indicate the $\mathrm{BD} /$ massive-planet companions discovered by Imaging, Transit, Radial Velocity, and Microlensing methods, respectively. The two solutions for OGLE2013-BLG-0911Lb are represented as stars.

stellar and planetary-mass companions (Marcy \& Butler 2000; Grether \& Lineweaver 2006; Johnson et al. 2010), the so-called "BD desert." It is likely that this BD deficit is because of differences between the formation mechanisms of companions with planetary mass and stellar mass. However, it is not yet clear if the BD-mass companions formed like planets in the protoplanetary disk, formed as binary stars in the molecular cloud, or were captured by the primary stars. Some theories have suggested that the BD desert might be an outcome of the interaction between massive companions and protoplanetary disks and/or of tidal evolution (Armitage \& Bonnell 2002; Matzner \& Levin 2005; Duchêne \& Kraus 2013).

Gravitational microlensing (Mao \& Paczynski 1991) surveys have probed the distribution of the outer planetary systems beyond the snow line (Hayashi 1981), where ice-dominated solid materials are rich, leading to efficient formation of gasgiant planets according to the core accretion theory (Lissauer 1993; Pollack et al. 1996). Because microlensing does not depend on the luminosity of the host star, the technique is sensitive to companions to low-mass objects such as late $\mathrm{M}$ dwarfs or even BDs. Furthermore, the host and any companions can still be inferred at distances all the way to the Galactic bulge. In contrast, the RV and transit (Borucki et al. 2010) methods, which have discovered the bulk of currently known exoplanets and BDs orbiting around hosts, have only a sensitivity to companions relatively close to hosts and whose hosts are sufficiently bright. Figure 1 shows the distribution of discovered $\mathrm{BD} /$ massive-planet companions around main-sequence stars and BDs. The RV (blue dots) and transit (green dots) methods have discovered a lot of the companions around $1 M_{\odot}$ stars but only a few around low-mass stars below $0.5 M_{\odot}$. This would be caused by an observational bias due to the faintness of low-mass stars in the visible wavelength range. The direct imaging (orange dots) method has detected the companions around hosts with masses of 0.01-3 $M_{\odot}$, but it could not have resolved the companions with relatively short orbital radii. On the other hand, microlensing (red dots) has discovered $\mathrm{BD} /$ massive-planet companions around hosts with masses of $\sim 0.05-1 M_{\odot}$ with orbital radii of $\sim 0.3-10$ au (e.g., Ranc et al. 2015; Han et al. 2017; Ryu et al.
2018), which are complementary to other detection methods. Gaudi (2002) estimated that more than $25 \%$ of BD companions with separations $\sim 1-10$ au would be detected by present microlensing surveys. According to the standard core accretion theory, massive planets as well as BDs are more difficult to form around low-mass $\mathrm{M}$ dwarfs than solar-type stars owing to low disk surface densities (Ida \& Lin 2005) and long timescales (Laughlin et al. 2004). It is possible to constrain the BD formation mechanism around late $\mathrm{M}$ dwarfs from a statistical analysis of microlensing results in the BD-mass regime, which can be compared to the lack of close-in BD companions around solar-type stars found by RV observations.

Shvartzvald et al. (2016, hereafter S16) conducted a statistical analysis of the first four seasons of a "secondgeneration" microlensing survey (Gaudi et al. 2009), which consisted of the observations by the Optical Gravitational Lensing Experiment (OGLE; Udalski et al. 1994) collaboration, the Microlensing Observations in Astrophysics (MOA; Bond et al. 2001; Sumi et al. 2003) collaboration, and the Wise team (Shvartzvald \& Maoz 2012). They analyzed 224 microlensing events and found 29 "anomalous" events, implying the presence of a companion to the lens host. They performed an automated coarse grid search for light-curve modelings rather than a detailed modeling of individual events for their statistical study. Finally, they derived the planet (binary) frequency distribution as a function of companion-tohost mass ratio $q$ and found a possible deficit at $q \sim 10^{-2}$. However, it is worthwhile to conduct a detailed analysis of individual "planetary candidate" in their sample, for which there are not any models in the literature. For example, they reported that OGLE-2013-BLG-0911 has a planetary massratio of $q \approx 3 \times 10^{-4}$, but we found new preferred solutions with a less extreme mass ratio, $q \approx 3 \times 10^{-2}$.

Here, we present the analysis of a high-magnification (maximum magnification of $A_{\max } \sim 220$ ) microlensing event, OGLE-2013-BLG-0911. The "anomaly", due to a companion to the lens star, was clearly detected near the peak of the light curve. We present the observations and data sets of the event in Section 2. Our light-curve analysis is described in Section 3. In Section 4, we present our analysis of the source 
properties. The physical parameters of the lens system are described in Section 5. We summarize and discuss the results in Section 6.

\section{Observation and Data Sets}

\subsection{Observation}

The microlensing event OGLE-2013-BLG-0911 was discovered and alerted as a microlensing candidate on 2013 June 3 UT 21:51 by the fourth phase of the OGLE collaboration (OGLE-IV; Udalski et al. 2015). OGLE-IV ${ }^{74}$ is conducting a microlensing exoplanet search toward the Galactic bulge using the $1.3 \mathrm{~m}$ Warsaw telescope of Las Campanas Observatory in Chile with a wide total field of view (FOV) of $1.4 \mathrm{deg}^{2}$. The OGLE observations were conducted using the standard $I-$ and near-standard $V$-band filters. The second phase of the MOA collaboration $^{75}$ (MOA-II; Bond et al. 2017) is also carrying out a microlensing survey toward the Galactic bulge using the $1.8 \mathrm{~m}$ MOA-II telescope with a $2.2 \mathrm{deg}^{2}$ FOV CCD camera (MOA-cam3; Sako et al. 2008) at Mount John Observatory in New Zealand. Thanks to its wide FOV, the MOA collaboration is observing bulge stars with a cadence of 15-90 minutes every day depending on the field. The MOA survey independently discovered and issued an alert for the event as MOA-2013BLG-551. The MOA observations were conducted using a custom wide-band filter, "MOA-Red," which corresponds approximately to the combination of the standard $I$ and $R$ filters. The Wise ${ }^{76}$ team also conducted a microlensing survey from 2010 to 2015 and monitored a field of $8 \mathrm{deg}^{2}$ within the observational footprints of both OGLE and MOA (Shvartzvald $\&$ Maoz 2012). They observed using the $1 \mathrm{~m}$ Wise telescope at Wise Observatory in Israel with a $1 \mathrm{deg}^{2}$ FOV LAIWO camera (Gorbikov et al. 2010) and the cadence for each of the eight Wise fields was $\sim 30$ minutes.

The event was located at (R.A., decl. $)_{\mathrm{J} 2000}=(17: 55: 31.98$, $-29: 15: 13.8)$ or Galactic coordinates $(l, b)=(0.84,-2.02)$. Real-time analysis predicted the event would reach high peak magnification during which the sensitivity to low-mass companions is high (Griest \& Safizadeh 1998; Rattenbury et al. 2002). Follow-up observations during the period of high magnification were encouraged to capture short planetary signals. Consequently, in addition to the OGLE and MOA survey observations, the light curve was densely observed by several follow-up groups: Microlensing Follow Up Network ( $\mu$ FUN; Gould et al. 2006), Microlensing Network for the Detection of Small Terrestrial Exoplanets (MiNDSTEp; Dominik et al. 2010), and RoboNet (Tsapras et al. 2009; Dominik et al. 2019). Hereafter, we refer this event as OGLE2013-BLG-0911.

\subsection{Data Reduction}

All of the data sets of OGLE-2013-BLG-0911 are summarized in Table 1. Most photometric pipelines use the Difference Image Analysis (DIA; Alard \& Lupton 1998; Alard 2000) technique, which is very effective in high stellar density fields such as those toward the Galactic bulge. The MOA and $\mu$ FUN CTIO data were reduced with the MOA implementation of the DIA method (Bond et al. 2001, 2017).

\footnotetext{
$\overline{74}$ http://ogle.astrouw.edu.pl/ogle4/ews/ews.html

75 https://www.massey.ac.nz/ iabond/moa/alerts/

76 http://wise-obs.tau.ac.il/ wingspan/
}

The OGLE data were reduced by OGLE's DIA pipeline (Wozniak 2000). The Wise data were reduced using the pySIS DIA software (Albrow et al. 2009). The other $\mu$ FUN data and MiNDSTEp data were reduced by DoPhot (Schechter et al. 1993) and DanDIA (Bramich 2008; Bramich et al. 2013). RoboNet data were reduced using a customized version of the DanDIA pipeline (Bramich 2008).

It is known that the nominal photometric error bars given by each photometric pipeline are potentially underestimated in high stellar density fields toward the bulge. Therefore, we empirically renormalized the error bars for each data set following procedure of Bennett et al. (2008) and Yee et al. (2012), i.e.,

$$
\sigma_{i}^{\prime}=k \sqrt{\sigma_{i}^{2}+e_{\min }^{2}}
$$

where $\sigma_{i}^{\prime}$ and $\sigma_{i}$ represent the renormalized errors and the original errors given by the pipelines, respectively. The parameters $k$ and $e_{\min }$ are the coefficients for the error renormalization. Here, $e_{\min }$ represents the systematic errors when the source flux is significantly magnified. We added $0.3 \%$ in quadrature to each error, i.e., $e_{\min }=0.003$, and then calculated $k$ values in order to achieve a value of $\chi^{2} /$ dof $=1$ for each data set (Bennett et al. 2014; Skowron et al. 2016). We list the renormalization coefficients $k$ in Table 1 along with the number of used data points $N_{\text {use }}$. We confirmed that the final best-fit model is consistent with the preliminary best-fit model found using the data sets before the error renormalization.

\section{Light-curve Modeling}

Here, we present the light-curve modeling for OGLE-2013BLG-0911. Figure 2 represents the light curve of OGLE-2013BLG-0911. The main anomalous feature can be seen between $6536.8<\mathrm{HJD}-245000<6537.6$. A standard single-lens single-source (1L1S) model fits the data worse than a binarylens single-source (2L1S) model by $\Delta \chi^{2}>3500$. In following sections, we present the details of the light-curve modeling for OGLE-2013-BLG-0911. In Table 2, we summarize the comparisons of the $\chi^{2}$, number of fitting parameters, and Bayesian information criterion (BIC) between the microlensing models we examined.

\subsection{Model Description}

Assuming a single source star, the observed flux at any given time in a microlensing event, $F_{\text {obs }}(t)$, can be modeled by the following equation:

$$
F_{\mathrm{obs}}(t)=A(t) F_{\mathrm{s}}+F_{\mathrm{b}},
$$

where $A(t)$ is the magnification of the source flux, $F_{\mathrm{s}}$ is the unmagnified source flux, and $F_{\mathrm{b}}$ is the blend flux. We note that $F_{\mathrm{s}}$ and $F_{\mathrm{b}}$ can be, during the fitting process, solved analytically by the linear Equation (2) at given $A(t)$. For a standard 1L1S model, there are four parameters that describe the light-curve features (Paczynski 1986): (1) the time of the source approaching closest to the lens center of mass, $t_{0}$, (2) the impact parameter, $u_{0}$, in units of the angular Einstein radius, $\theta_{\mathrm{E}}$, (3) the Einstein radius crossing time, $t_{\mathrm{E}}$, and (4) the source angular radius, $\rho$, in units of $\theta_{\mathrm{E}}$. The measurement of $\rho$ is important because it leads to a determination of $\theta_{\mathrm{E}}$, which is 
Table 1

Data Sets for OGLE-2013-BLG-0911

\begin{tabular}{|c|c|c|c|c|c|c|}
\hline Site & Telescope & Collaboration & Label & Filter & $N_{\text {use }}$ & $k^{\mathrm{a}}$ \\
\hline Mount John Observatory & MOA-II $1.8 \mathrm{~m}$ & MOA & MOA & Red & 8761 & 1.055 \\
\hline Las Campanas Observatory & Warsaw $1.3 \mathrm{~m}$ & OGLE & OGLE & $I$ & 6895 & 1.480 \\
\hline Las Campanas Observatory & Warsaw $1.3 \mathrm{~m}$ & OGLE & OGLE & $V$ & 78 & 1.344 \\
\hline Florence and George Wise Observatory & Wise $1 \mathrm{~m}$ & Wise & Wise $1 \mathrm{~m}$ & $I$ & 253 & 0.947 \\
\hline Cerro Tololo-Inter-American Observatory (CTIO) & SMARTS $1.3 \mathrm{~m}$ & $\mu \mathrm{FUN}$ & CT13 & $I$ & 189 & 1.230 \\
\hline Cerro Tololo-Inter-American Observatory (CTIO) & SMARTS $1.3 \mathrm{~m}$ & $\mu \mathrm{FUN}$ & CT13 & $V$ & 35 & 1.182 \\
\hline Farm Cove Observatory & Farm Cove $0.36 \mathrm{~m}$ & $\mu \mathrm{FUN}$ & $\mathrm{FCO}$ & Unfiltered & 55 & 2.146 \\
\hline Weizmann Institute of Science, Marty S. Kraar Observatory & Weizmann 16 inch & $\mu \mathrm{FUN}$ & WIS & $I$ & 17 & 1.140 \\
\hline Haleakala Observatory & Faulkes North $2.0 \mathrm{~m}$ & RoboNet & FTN & $i^{\prime}$ & 27 & 2.181 \\
\hline Siding Spring Observatory (SSO) & LCO $1.0 \mathrm{~m}$, Dome A & RoboNet & $\operatorname{cojA}$ & $i^{\prime}$ & 31 & 1.920 \\
\hline Cerro Tololo Inter-American Observatory (CTIO) & LCO $1.0 \mathrm{~m}$, Dome B & RoboNet & lscB & $i^{\prime}$ & 51 & 1.311 \\
\hline Cerro Tololo Inter-American Observatory (CTIO) & LCO $1.0 \mathrm{~m}$, Dome C & RoboNet & lscC & $i^{\prime}$ & 71 & 2.315 \\
\hline South African Astronomical Observatory (SAAO) & LCO $1.0 \mathrm{~m}$, Dome A & RoboNet & cptA & $i^{\prime}$ & 32 & 0.559 \\
\hline South African Astronomical Observatory (SAAO) & LCO $1.0 \mathrm{~m}$, Dome B & RoboNet & cptB & $i^{\prime}$ & 8 & 0.497 \\
\hline ESO’s La Silla Observatory & Danish $1.54 \mathrm{~m}$ & MiNDSTEp & Dan & $I$ & 76 & 2.087 \\
\hline Salerno University Observatory & Salerno $0.36 \mathrm{~m}$ & MiNDSTEp & Sal & $I$ & 20 & 1.607 \\
\hline
\end{tabular}

Notes. The WIS, Sal, and lscC data are binned for 0.01 days.

${ }^{\mathrm{a}}$ The coefficient for error renormalization, see the text.

needed for the determination of the the mass-distance relation of the lens system.

In our fitting process, we used a Markov Chain Monte Carlo (MCMC) method (Verde et al. 2003) combined with our implementation of the inverse ray-shooting method (Bennett \& Rhie 1996; Bennett 2010) in order to find the best-fit model and estimate the parameter uncertainties from MCMC stationary distribution for each parameter. Linear limb-darkening models were used to describe the source star(s) in this work. From the measurement of the intrinsic source color of $(V-I)_{s, 0}=0.71$ described in Section 4, we assumed an effective temperature $T_{\text {eff }}=5750 \mathrm{~K}$ (González Hernández \& Bonifacio 2009), surface gravity $\log g=4.5$, and metallicity $\log [M / H]=0$. According to the ATLAS model of Claret \& Bloemen (2011), we selected limb-darkening coefficients of $u_{\mathrm{Red}}=$ $0.5900, u_{I}=0.5493, u_{V}=0.7107$. Here, $u_{\text {Red }}$ for the MOARed band is estimated as the mean of $u_{I}$ and $u_{R}$, and the $R$-band coefficient $u_{R}=0.6345$ is used for an unfiltered band.

\subsection{Binary-lens (2L1S) Model}

For a standard 2L1S model, there are three additional parameters: (1) the lens mass ratio between the host and a companion, $q$, (2) the projected binary separation in unit of the Einstein radius, $s$, and (3) the angle between the source trajectory and the binary-lens axis, $\alpha$. Here, we introduce two fitting parameters $t_{c}$ and $u_{c}$, for wide $(s>1)$ models. If $s>1$, the system center in our numerical code is offset from the binary center of mass by

$$
\Delta(x, y)=\left[\frac{q}{1+q}\left(\frac{1}{s}-s\right), 0\right]
$$

where $(x, y)$ are the parallel and vertical coordinate axes to the binary-lens axis on the lens plane (Skowron et al. 2011), and then we define the time of the source approaching closest to the "system center" and the impact parameter in units of the angular Einstein radius as $t_{c}$ and $u_{c}$, respectively.

\subsubsection{Static Models}

At first, we explored the 2L1S interpretation to explain the anomalous features of the light curve. In modeling $2 \mathrm{~L} 1 \mathrm{~S}$ microlensing light curves, it is common to encounter situations where different physical models explain the observed data equally well, e.g., the close/wide degeneracy (Griest \& Safizadeh 1998; Dominik 1999) and the planet/binary degeneracy (Choi et al. 2012; Miyazaki et al. 2018), where different combinations of the microlensing parameters can generate morphologically similar light curves. Therefore, we should thoroughly investigate the multi-dimensional parameter space to find the global preferred model solution. We conducted a detailed grid search over the $(q, s, \alpha)$ parameter space where the magnification pattern strongly depends on these three parameters. The search ranges of $q, s$, and $\alpha$ are $-1<\log s<1,-4.5<\log q<0$, and $0<\alpha<2 \pi$ with 40 grid points, respectively, and thus, the total number of grid points is $40 \times 40 \times 40=64,000$. We conducted the grid search analysis following the same procedure written in Miyazaki et al. (2018). Figure 3 shows the map of the minimum $\Delta \chi^{2}$ in each $s-q$ grid from the grid search. In Figure 3, we found two possible local minima around $(\log q$, $\log s) \sim(-1.8,-0.75)$ and $\sim(-1.8,0.75)$, which is caused by the close/wide degeneracy. After refining all possible solutions, we found the best-fit 2L1S close $(s<1)$ and wide $(s>1)$ models with $q \sim 0.03$, where the $\chi^{2}$ difference between them is only $\Delta \chi^{2}=4.9$. As seen in Figure 2 , the $2 \mathrm{~L} 1 \mathrm{~S}$ model with $q \sim 0.03$ provide good fits to the anomalous features around the top of the light curve. We also show the model light curve of 2L1S with $q \sim 10^{-4}$ in Figure 2, and it does not fit the lightcurve anomaly well.

S16 included this event in their statistical analysis as a planetary microlensing event, using a mass ratio of $q \sim 10^{-4}$ for this event. However, our reanalysis found that the static 2L1S models with $q \sim 10^{-2}$ are preferred over the model with $q \sim 10^{-4}$ by $\Delta \chi^{2}>700$. The reason for this oversight is that models with $q \sim 10^{-2}$ are outside of the range of their grid search of $-6<\log q<0$ and $0.3<s<3$, and the search for the best-fit model outside of this range by refining model parameters found by their grid search was not conducted. 

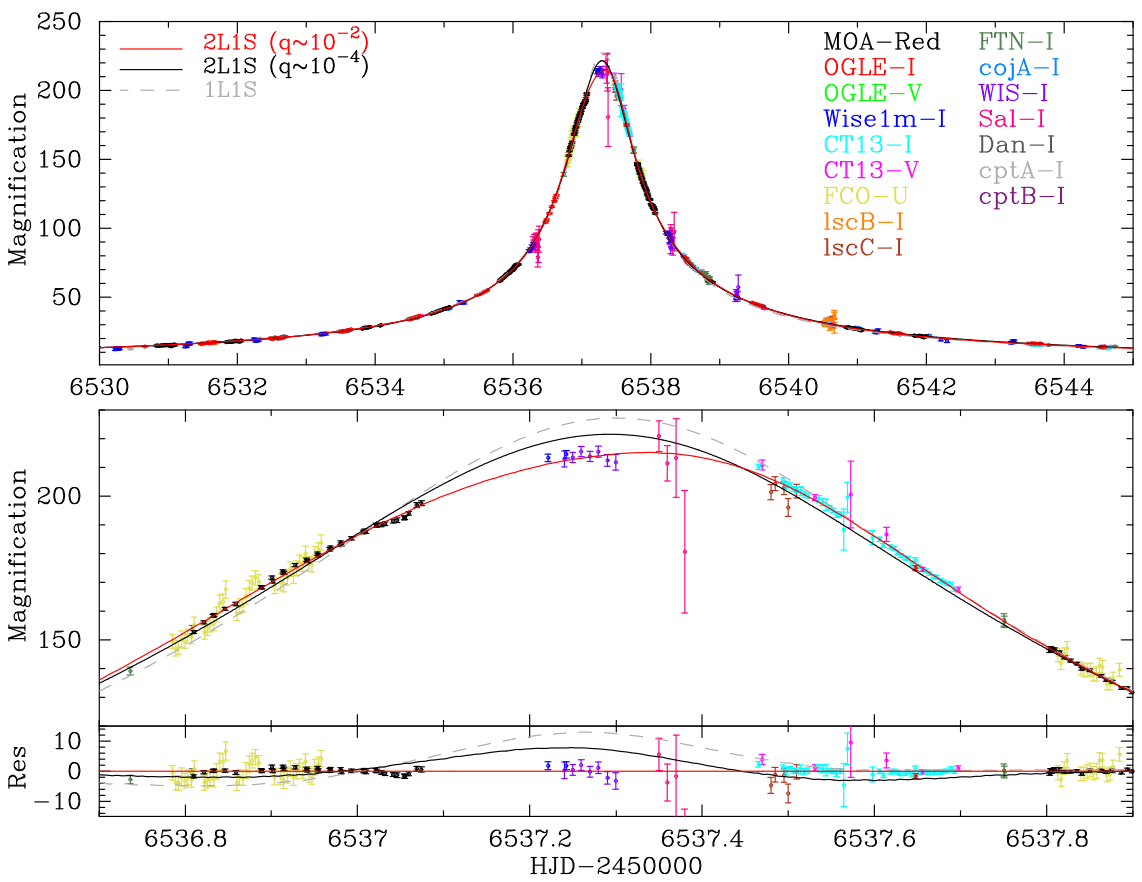

Figure 2. (Top panel) Light curve of OGLE-2013-BLG-0911. Each color on the data point corresponds to each instrument, shown on the right. The error bars are renormalized following Equation (1). The solid red, solid black, and dashed gray curves represent the static $2 \mathrm{~L} 1 \mathrm{~S}$ with $q \sim 10^{-2}$, 2L1S with $q \sim 10^{-4}$, and $1 \mathrm{~L} 1 \mathrm{~S}$ models, respectively. (Middle panel) A zoom-in around the peak. (Bottom panel) Residuals of the zoom-in light curve from the model of $2 \mathrm{~L} 1 \mathrm{~S}$ with $q \sim 10^{-2}$.

Another difference from S16 is that we used re-reduced MOA and OGLE light curves and included all of the follow-up data sets. However, we confirmed that the 2L1S models with $q \sim 10^{-4}$ are disfavored relative to the models with $q \sim 10^{-2}$ by $\Delta \chi^{2}>300$ even if we used the survey data, MOA, OGLE, and Wise $1 \mathrm{~m}$. Therefore, note that the survey data were sufficient to identify the new solutions.

\subsubsection{Parallax Effects}

Although the best-fit static models provide good fits to the main anomaly features around the peak of the light curve, we found that, overall, the light curve slightly deviates from the static models. The event OGLE-2013-BLG-0911 has $t_{\mathrm{E}} \sim 90$ days and had continued throughout the bulk of the bulge season, which implies that the light curve could be affected by additional high-order microlensing effects.

It is known that the orbital acceleration of Earth causes a parallax effect (Gould 1992, 2004; Smith et al. 2003). This can be described by the microlensing parallax vector $\pi_{\mathrm{E}}=$ $\left(\pi_{\mathrm{E}, N}, \pi_{\mathrm{E}, E}\right)$. Here, $\pi_{\mathrm{E}, N}$ and $\pi_{\mathrm{E}, E}$ denote the north and east components of $\pi_{\mathrm{E}}$ projected to the sky plane in equatorial coordinates. The direction of $\pi_{\mathrm{E}}$ is defined so as to be identical to that of $\boldsymbol{\mu}_{\text {rel,G }}$, which is the geocentric lens-source relative proper motion projected to the sky plane at a reference time $t_{\text {fix }}$, and the amplitude of $\pi_{\mathrm{E}}$ is $\pi_{\mathrm{E}}=\mathrm{au} / \tilde{r}_{\mathrm{E}}$ where $\tilde{r}_{\mathrm{E}}$ is the Einstein radius projected inversely to the observer plane. We took a reference time $t_{\text {fix }}=6537.3$ days for this event. The measurement of $\pi_{\mathrm{E}}$ enables constraints to be placed on the relation between the lens mass $M_{L}$ and distance $D_{L}$ (Gould 2000; Bennett 2008). For Galactic bulge source events, models with $\left(u_{0}, \alpha, \pi_{\mathrm{E}, N}\right)$ and $-\left(u_{0}, \alpha, \pi_{\mathrm{E}, N}\right)$ can yield very similar light curves (Skowron et al. 2011). This is reflected as a pair of the symmetric source trajectories to the binary and is sometimes referred to as "ecliptic degeneracy."
Taking the parallax effect into consideration for modeling, we found that the two parallax parameters gave an improvement of $\Delta \chi^{2} \sim 210$ compared to the best-fit static model. However, we also found that the best-fit parallax model does not seem to explain the long-term deviations of the light curve from the best-fit static model, as can be seen in Figure 4. This implies that there might still be other high-order microlensing effects in the light curve. Note that adding the lens orbital motion does not improve our models.

\subsubsection{Xallarap Effects}

Xallarap (Griest \& Hu 1992; Han \& Gould 1997; Poindexter et al. 2005) is the microlensing effect on the light curve induced by the source orbital motion around the source companion. The xallarap model requires seven additional fitting parameters that determine the orbital elements of the source system; the direction toward the solar system relative to the orbital plane of the source system, R.A. $\xi$ and decl. $\xi$; the source orbital period, $P_{\xi}$; the source orbital eccentricity and perihelion time, $e_{\xi}$ and $T_{\text {peri }}$; the xallarap vector, $\boldsymbol{\xi}_{\mathrm{E}}=\left(\xi_{\mathrm{E}, N}, \xi_{\mathrm{E}, E}\right)$. The direction of $\boldsymbol{\xi}_{\mathrm{E}}$ is similar to that of the geocentric lens-source proper motion $\boldsymbol{\mu}_{\text {rel,G }}$ and the amplitude of $\boldsymbol{\xi}_{\mathrm{E}}$ is $\xi_{\mathrm{E}}=a_{S} / \hat{r}_{\mathrm{E}}$ where $a_{S}$ is the semimajor axis of the source orbit and $\hat{r}_{\mathrm{E}}$ is the projected Einstein radius to the source plane, i.e., $\hat{r}_{\mathrm{E}}=\theta_{\mathrm{E}} D_{S}$. Kepler's third and Newton's third laws give the following relations (Batista et al. 2009):

$$
\begin{gathered}
\xi_{\mathrm{E}}=\frac{1 \mathrm{au}}{D_{S} \theta_{\mathrm{E}}}\left(\frac{M_{c}}{M_{\odot}}\right)\left[\frac{M_{\odot}}{M_{c}+M_{S}} \frac{P_{\xi}}{1 \mathrm{yr}}\right]^{2 / 3}, \\
M_{S} a_{S}=M_{C} a_{C} \Rightarrow a_{\mathrm{SC}} \equiv a_{S}+a_{C}=\left(1+\frac{M_{S}}{M_{C}}\right) a_{S},
\end{gathered}
$$

where $M_{S}$ and $M_{C}$ are the masses of the source and source companion, respectively. Therefore, we can estimate the source 
Table 2

Comparisons between Each Microlensing Model

\begin{tabular}{|c|c|c|c|c|c|c|c|}
\hline \multicolumn{3}{|c|}{ Model } & $N_{\text {param }}{ }^{\mathrm{a}}$ & $\chi^{2}$ & $\mathrm{BIC}^{\mathrm{b}}$ & $\Delta \chi^{2}$ & $\triangle \mathrm{BIC}$ \\
\hline $1 \mathrm{~L} 1 \mathrm{~S}$ & $\cdots$ & Static & 4 & 21027.4 & 21066.3 & 4485.1 & 4368.5 \\
\hline $1 \mathrm{~L} 2 \mathrm{~S}$ & $\cdots$ & Static & 10 & 18631.0 & 18728.2 & 2088.7 & 2030.4 \\
\hline $2 \mathrm{~L} 1 \mathrm{~S}$ & $(s<1)$ & Static & 7 & 17473.7 & 17541.7 & 931.4 & 843.9 \\
\hline 2L1S & $\left(s<1, u_{0}>0\right)$ & Parallax & 9 & 17262.7 & 17350.2 & 720.4 & 652.4 \\
\hline $2 \mathrm{~L} 1 \mathrm{~S}$ & $\left(s<1, u_{0}>0\right)$ & Xallarap & 14 & 16587.6 & 16723.6 & 45.3 & 25.8 \\
\hline $2 \mathrm{~L} 2 \mathrm{~S}$ & $\left(s<1, u_{0}>0\right)$ & Parallax +Xallarap & 16 & 16542.3 & 16697.8 & $\cdots$ & $\cdots$ \\
\hline
\end{tabular}

Notes.

a Number of fitting parameters.

${ }^{b} \mathrm{BIC}$.

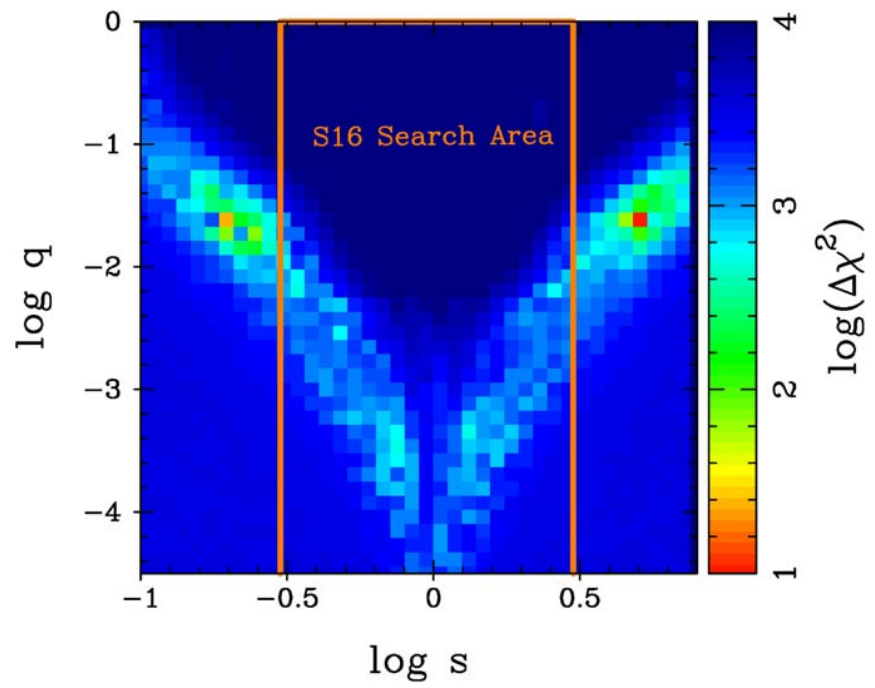

Figure 3. Map of the minimum $\Delta \chi^{2}$ in each $s-q$ grid from the grid search. The orange box corresponds to the area of the grid search analysis in Shvartzvald et al. (2016).

companion mass $M_{C}$ from the xallarap measurements by assuming $M_{S}$ and $D_{S}$.

Since the number of additional parameters for the xallarap effect is large, we conducted a grid search fixing (R.A. $\xi$, $\operatorname{decl}_{\xi}, P_{\xi}$ ) in order to avoid missing any local minima. After refining all possible solutions, we found that the best-fit xallarap model is favored over the best-fit parallax model by $\Delta \chi^{2}>650$. As shown in Figure 4, including the xallarap effect produces a model that fits the long-term residuals from the best-fit static model, and it dramatically improves the $\chi^{2}$ values. The best-fit orbital period of the source system is $P_{\xi} \sim 40$ days and is clearly different from Earth's orbital period of 365 days, which implies that the parallax and xallarap signals are clearly distinguishable. Following Equation (3), the best-fit 2L1S xallarap model indicates a source companion mass of $M_{C}=0.21 M_{\odot}$ and a distance between two sources $a_{\mathrm{SC}}=0.22 \mathrm{au}$ on the assumption of $M_{S}=1.0 M_{\odot}$ and $D_{S}=$ $8 \mathrm{kpc}$, which is a common stellar binary system in solar neighborhood (Duchêne \& Kraus 2013). The best-fit $\xi_{\mathrm{E}}$ values are much smaller than 1 , which means that the two sources are separated by much less than the Einstein radius. Hence, the source companion was also likely to be magnified during the event. In the following sections, we explore binary source scenarios where both components of the binary source system are magnified by the lens.

\subsection{Binary Source (1L2S) Model}

When two source stars are magnified by the same singlelens, called a 1L2S event, the observed flux would be the superposition of the two magnified single-source fluxes, i.e.,

$$
A(t)=\frac{A_{1}(t) F_{s, 1}+A_{2}(t) F_{s, 2}}{F_{s, 1}+F_{s, 2}}=\frac{A_{1}(t)+q_{F, j} A_{2}(t)}{1+q_{F, j}},
$$

where $A_{i}$ and $F_{s, i}$ represent the magnification and the baseline flux of each $i$-th source, and $q_{F, j}=F_{s, 2} / F_{s, 1}$ is the flux ratio between the two source stars in each $j$-th passband. For a standard (static) 1L2S model, the fitting parameters are $\left[t_{0}, t_{0,2}\right.$, $\left.t_{\mathrm{E}}, u_{0}, u_{0,2}, \rho, \rho_{2}, q_{F, j}\right]$. Because the magnification of each source star varies independently, the total observed source color is variable during a binary source event, which happens in single-source events only if limb-darkening effects are seen during caustic crossings ${ }^{77}$, as microlensing does not depend on wavelength. Binary source events can mimic short-term binarylens anomalies in a light curve; therefore, it is necessary to determine whether the anomaly features are induced by the binary lens or binary source (Gaudi 1998; Jung et al. 2017a, 2017b; Shin et al. 2019).

First, we fitted the light curves with the static 1L2S model and found that it was disfavored over the static $2 \mathrm{~L} 1 \mathrm{~S}$ models by $\Delta \chi^{2}>1100$. In Section 3.2.3, we found an asymmetric distortion in the light curves that can be explained by the xallarap effect (i.e., source orbital effect). Thus, we also explored 1L2S models with source orbital motion. The trajectories of two sources can be estimated by the source orbital motion from the xallarap parameters, $\left(\xi_{\mathrm{E}, N}, \xi_{\mathrm{E}, E}\right.$, R.A. $\xi$, decl. $\left.\xi, P_{\xi}, e_{\xi}, T_{\mathrm{peri}}\right)$, and Equation (3). Here, we assumed $M_{S}=1 M_{\odot}$ and $D_{S}=8 \mathrm{kpc}$ to derive the source companion mass $M_{C}$. In Appendix B, we confirmed that the assumptions of $M_{S}=1 M_{\odot}$ and $D_{S}=8 \mathrm{kpc}$ hardly impact the light-curve modeling. We conducted a detailed grid search of (R.A. $\xi$ decl. $_{\xi}, P_{\xi}$ ) and refined all possible 1L2S solutions. We found that the best-fit $1 \mathrm{~L} 2 \mathrm{~S}$ model is not preferred over the

\footnotetext{
77 For point lenses, this happens only if the lens briefly transits the source (Loeb \& Sasselov 1995; Gould \& Welch 1996).
} 


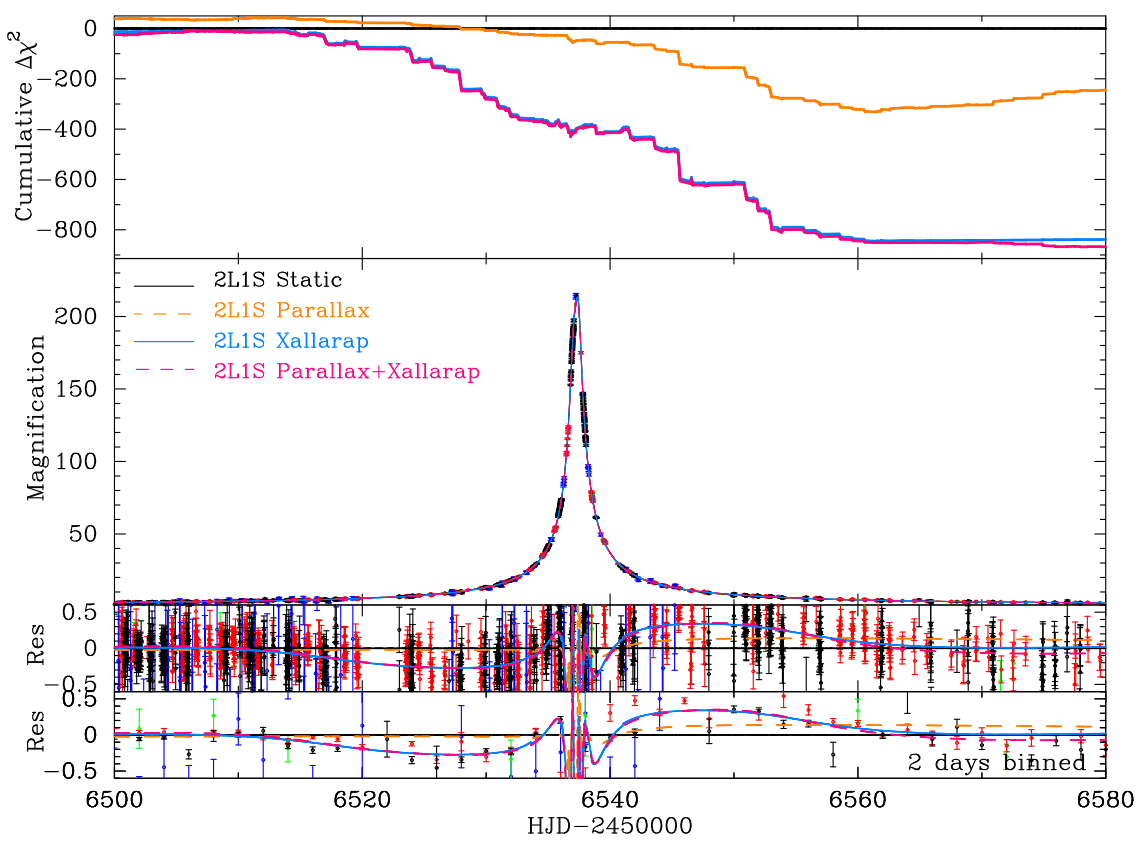

Figure 4. (Top panel) Cumulative $\Delta \chi^{2}$ distributions of the three 2L1S close $\left(u_{0}>0\right)$ models compared to the 2L1S static model. (Second panel from the top) The light curve and models for OGLE-2013-BLG-0911. Here, we plot only MOA, OGLE, and Wise 1m light curves for clarity. (Third panel from the top) The residuals of the light curve and models from the static model. (Bottom panel) The residuals binned by 2 days.

static 2 L1S models by $\Delta \chi^{2}>80$, even if we introduced the source orbital motion.

\subsection{Binary-lens Binary-source (2L2S) Model}

Finally, we explored the 2L2S models with source orbital motion, i.e., taking into account the flux from the source companion and the xallarap effect. Here, we adopted the flux ratio $q_{F}$ estimated from $M_{C}$, which is derived from the xallarap parameters to maintain consistency. We derived the flux ratios in each band from a combination of $M_{C}$ and a theoretical stellar isochrone model $^{78}$ (PARSEC; Bressan et al. 2012) for solar metallicity and a typical bulge star age of $10 \mathrm{Gyr}$. For the MOA-Red band, we derived the flux ratio from that in $I$ - and $V$ - bands, $q_{F, \text { Red }}=q_{F, I}^{0.827} q_{F, V}^{0.173}$. This formula comes from the following color transformation that is derived by using bright stars around the event (Gould et al. 2010; Bennett et al. 2012, 2018),

$$
R_{\mathrm{MOA}}-I_{\mathrm{O} 3}=0.173\left(V_{\mathrm{O} 3}-I_{\mathrm{O} 3}\right)+\mathrm{const}
$$

where $R_{\mathrm{MOA}}, I_{\mathrm{O} 3}$, and $V_{\mathrm{O} 3}$ are the magnitudes in MOARed, OGLE-III $I$-, and $V$-bands, respectively. For the Unfiltered passband, we used the $R$-band flux ratio assuming $q_{F, \text { Unfiltered }} \approx q_{F, R}$.

We found the four best $2 \mathrm{~L} 2 \mathrm{~S}$ models, which suffer from the close/wide degeneracy and the ecliptic degeneracy. The parameters of these models are shown in Table 3. The light curve of the best-fit $2 \mathrm{~L} 2 \mathrm{~S}\left(s<1, u_{0}>0\right)$ model is shown in Figure 5. Here, as shown in Equation (5), the light curves in each passband are different. The black, red, green, and cyan solid curves indicate the model light curves in the MOA-Red, $I-, V$-, and $R$-bands, respectively. The caustic geometry and source trajectories of the best-fit $2 \mathrm{~L} 2 \mathrm{~S}\left(s<1, u_{0}>0\right)$ model are shown in Figure 6. Here, the source companion trajectory indicates that the source companion is more strongly magnified

\footnotetext{
78 http://stev.oapd.inaf.it/cgi-bin/cmd
}

than the primary source. In general, such magnification differences in two sources allow us to resolve the close/wide degeneracy and the ecliptic degeneracy. However, as shown in the bottom right panel of Figure 5, where the secondary source magnification is peaked at HJD' $\sim 6539.55$, the flux contribution is $\sim 0.01$ times smaller than the primary source because the source companion is intrinsically much fainter than the primary source. Consequently, we could not resolve these degeneracies. These $2 \mathrm{~L} 2 \mathrm{~S}$ models are preferred relative to the 2L1S models with parallax and xallarap effects by $\Delta \chi^{2} \sim 16$ without additional fitting parameters. The fitting and physical parameters for the $2 \mathrm{~L} 1 \mathrm{~S}$ and $2 \mathrm{~L} 2 \mathrm{~S}$ models are almost identical each other. Therefore, it hardly affects the final results, whichever we take. Hereafter, we take the $2 \mathrm{~L} 2 \mathrm{~S}$ models for the final result.

\section{Source Properties}

The measurement of $\rho$ enables us to determine the angular Einstein radius $\theta_{\mathrm{E}}=\theta_{*} / \rho$ where $\theta_{*}$ is the angular source radius. The angular source radius $\theta_{*}$ can be estimated from the extinction-free color and magnitude of the source star by using a method similar to that of Yoo et al. (2004), which adopts the centroid of the bulge red clump giants (RCG) as a reference point. Yoo et al. (2004) assumed that the source star suffers from the same extinction as that of the bulge RCG so that the extinction-free color and magnitude of the source star can be described by the following equation:

$$
(V-I, I)_{S, 0}=(V-I, I)_{0, \mathrm{RCG}}-\Delta(V-I, I),
$$

where $(V-I, I)_{0, \mathrm{RCG}}=(1.06 \pm 0.07,14.40 \pm 0.04)$ is the extinction-free color and magnitude of the bulge RCG centroid (Bensby et al. 2011, 2013; Nataf et al. 2013) and $\Delta(V-I, I)$ are the offsets of the color and magnitude from the RCG centroid to the source star measured in the standard colormagnitude diagram (CMD). 
Table 3

2L2S Model Parameters

\begin{tabular}{|c|c|c|c|c|c|}
\hline \multirow{2}{*}{ Parameters } & \multirow{2}{*}{ Units } & \multicolumn{2}{|c|}{ Close $(s<1)$} & \multicolumn{2}{|c|}{ Wide $(s>1)$} \\
\hline & & $\left(u_{0}>0\right)$ & $\left(u_{0}<0\right)$ & $\left(u_{c}>0\right)$ & $\left(u_{c}<0\right)$ \\
\hline$t_{0}\left(t_{c}\right)$ & HJD-2456530 & $7.3128_{-0.0005}^{+0.0005}$ & $7.3127_{-0.0005}^{+0.0005}$ & $7.3123_{-0.0004}^{+0.0003}$ & $7.3111_{-0.0006}^{+0.000}$ \\
\hline$u_{0}\left(u_{c}\right)$ & $\left(10^{-3}\right)$ & $4.800_{-0.079}^{+0.077}$ & $-4.620_{-0.042}^{+0.041}$ & $4.522_{-0.108}^{+0.086}$ & $-4.626_{-0.053}^{+0.053}$ \\
\hline$q$ & $\left(10^{-2}\right)$ & $3.236_{-0.084}^{+0.089}$ & $3.066_{-0.094}^{+0.090}$ & $3.160_{-0.134}^{+0.132}$ & $3.456_{-0.067}^{+0.087}$ \\
\hline$s$ & $\ldots$ & $0.150_{-0.002}^{+0.002}$ & $0.150_{-0.002}^{+0.002}$ & $6.774_{-0.085}^{+0.101}$ & $7.084_{-0.064}^{+0.074}$ \\
\hline$\pi_{\mathrm{E}, N}$ & $\ldots$ & $0.256_{-0.050}^{+0.044}$ & $0.300_{-0.025}^{+0.027}$ & $0.319_{-0.050}^{+0.039}$ & $0.271_{-0.041}^{+0.032}$ \\
\hline$\pi_{\mathrm{E}, E}$ & $\ldots$ & $0.018_{-0.005}^{+0.005}$ & $0.004_{-0.006}^{+0.006}$ & $0.001_{-0.006}^{+0.006}$ & $0.006_{-0.004}^{+0.003}$ \\
\hline$\xi_{\mathrm{E}, N}$ & $\left(10^{-3}\right)$ & $-2.91_{-1.01}^{+0.97}$ & $-3.13_{-1.39}^{+1.72}$ & $-5.32_{-1.61}^{+2.66}$ & $-2.48_{-1.04}^{+1.28}$ \\
\hline$\xi_{\mathrm{E}, E}$ & $\left(10^{-3}\right)$ & $-4.31_{-0.17}^{+0.18}$ & $-3.59_{-0.44}^{+0.62}$ & $-3.53_{-0.48}^{+0.45}$ & $-3.66_{-0.33}^{+0.50}$ \\
\hline R.A. $\xi$ & degree & $-74.2_{-12.2}^{+12.3}$ & $-87.4_{-14.0}^{+15.5}$ & $260.9_{-11.4}^{+13.5}$ & $-89.4_{-16.3}^{+16.3}$ \\
\hline$q_{F, \text { Red }}$ & $\left(10^{-3}\right)$ & $1.122_{-0.330}^{+0.388}$ & $0.818_{-0.254}^{+0.317}$ & $0.974_{-0.298}^{+0.439}$ & $0.970_{-0.261}^{+0.342}$ \\
\hline$q_{F, I}$ & $\left(10^{-3}\right)$ & $1.424_{-0.399}^{+0.466}$ & $1.058_{-0.309}^{+0.383}$ & $1.246_{-0.362}^{+0.527}$ & $1.241_{-0.317}^{+0.412}$ \\
\hline$q_{F, V}$ & $\left(10^{-4}\right)$ & $3.58_{-1.29}^{+1.58}$ & $2.39_{-0.93}^{+1.24}$ & $3.00_{-1.12}^{+1.76}$ & $2.98_{-0.99}^{+1.36}$ \\
\hline$q_{F, R}$ & $\left(10^{-4}\right)$ & $6.62_{-2.18}^{+2.64}$ & $4.61_{-1.62}^{+2.10}$ & $5.63_{-1.93}^{+2.96}$ & $5.60_{-1.70}^{+2.30}$ \\
\hline$\pi_{\mathrm{E}}$ & $\ldots$ & $0.257_{-0.050}^{+0.044}$ & $0.300_{-0.025}^{+0.027}$ & $0.319_{-0.050}^{+0.039}$ & $0.271_{-0.041}^{+0.032}$ \\
\hline$\chi^{2}$ & $\ldots$ & 16542.2 & 16543.3 & 16542.3 & 16542.9 \\
\hline$\Delta \chi^{2}$ & $\ldots$ & $\ldots$ & 1.1 & 0.1 & 0.7 \\
\hline
\end{tabular}

Note. Here, we assume $M_{S}=1 M_{\odot}$ and $D_{S}=8 \mathrm{kpc}$. The flux ratios $q_{F}$ and parallax amplitude $\pi_{\mathrm{E}}=\sqrt{\pi_{\mathrm{E}, \mathrm{N}}^{2}+\pi_{\mathrm{E}, \mathrm{E}}^{2}}$ are not fitting parameters. All other parameters in this table are used as fitting parameters for modeling.

\subsection{Photometric Source Properties}

We obtained the apparent source color and magnitude of $(V-I, I)_{S}=(1.904 \pm 0.008,19.618 \pm 0.006)$ derived from the measurements of CT13-I and $V$ in the light-curve modeling, which is detailed in Appendix A. We also derived the source color and magnitude from the measurements of OGLE- $I$ and $V$ and confirmed that they are consistent within $2 \sigma$, which is also detailed in Appendix A. In addition, we independently measured the source color using a linear regression from CT13-I and $V,(V-I)_{\mathrm{CT} 13 \text {,reg }}=1.910 \pm 0.005$, which is consistent with $(V-I)_{S}$. Therefore, we determined that the measurements of the source color and magnitude are robust. Here, we took the source color and magnitude derived from the CT-13 measurements because both CT13- $I$ and $-V$ covered the light curve well when the primary source were significantly magnified.

Figure 7 shows the CMD of the OGLE-III catalog within $60^{\prime}$ of the sources plotted as black dots, and the CMD of Baade's window from Holtzman et al. (1998) plotted as green dots. We found that the extinction-free color and magnitude of the primary source star are $(V-I, I)_{S, 0}=(0.582 \pm 0.071,17.936 \pm 0.049)$ assuming that the source suffers from the same extinction of the RCG centroid of $\left(E(V-I), A_{I}\right)_{\mathrm{RCG}}=(1.322 \pm 0.071,1.682 \pm$ $0.049)$. The primary and secondary source stars are represented as the blue and magenta dots in Figure 7. The primary source star seems to be somewhat bluer and brighter than other typical bulge dwarfs, which implies that the source possibly suffered less from reddening and extinction than the bulge RCG centroid.

\subsection{Spectroscopic Source Properties}

Bensby et al. (2017) took a spectrum of OGLE-2013-BLG0911S and reported the source properties in detail, which are summarized in Table $4{ }^{79}$ They suggested a possibility that the source star belongs to the foreground Galactic disk for three reasons. First, they measured the lens-source relative proper

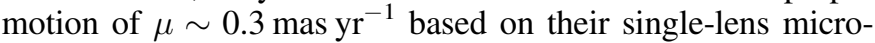
lensing model and indicated that this small value preferred the foreground disk source. Second, the intrinsic source color $(V-I)_{S, 0}=0.71_{-0.02}^{+0.03}$ based on their spectroscopic measurement is redder than $(V-I)_{S, 0}=0.49$ from their microlensing analysis, which implies that the source suffers less extinction than the average RCG in this field. They suggested that this may be because the source is in the foreground disk. Note that our derived $(V-I)_{S, 0}$ assuming the source is behind all of the dust, is less blue (0.58 versus 0.49$)$, but is still substantially bluer than Bensby's spectroscopic value. Third, they claimed that the heliocentric $\mathrm{RV}$ of the source, $\mathrm{RV}_{\text {helio, }}$, is consistent with a disk star.

However, if we adopt $I_{S, 0}=17.94$, which is derived from our light-curve modeling and the absolute source magnitude $M_{I}=2.98$, which is estimated from spectroscopic values in Bensby et al. (2017), these measurements yield a source distance of $\sim 9.8 \mathrm{kpc}$, which would put the source within or behind the bulge. Furthermore, we consider that the above rationale for the disk source scenario is not strong for three

\footnotetext{
${ }^{79}$ http://cdsarc.u-strasbg.fr/viz-bin/qcat?J/A+A/605/A89
} 

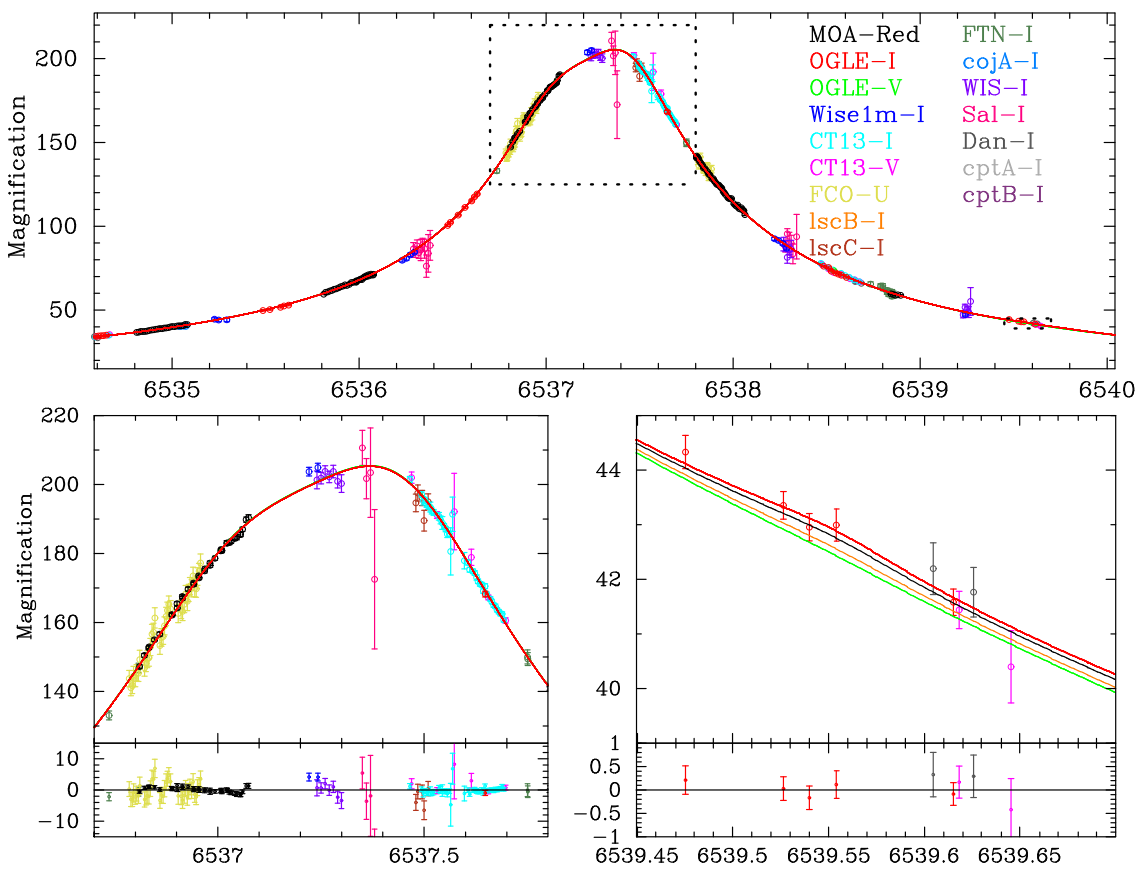

Figure 5. Light curve of OGLE-2013-BLG-0911. Each color on the data point corresponds to each instrument, shown on the right. The error bars are renormalized following Equation (1). The 2L2S $\left(s<1, u_{0}>0\right)$ model light curves in MOA-Red, $I$, $V$, Unfiltered bands are shown as the solid black, red, green, and orange lines, respectively. The dotted boxes in the top panel correspond to the areas represented in the bottom left and right panels, where the primary and secondary sources were significantly magnified, respectively.

reasons. First, both our $2 \mathrm{~L} 2 \mathrm{~S}$ and $2 \mathrm{~L} 1 \mathrm{~S}$ models provided

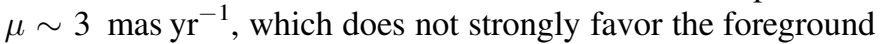
disk source. It is likely that their 1L1S model, which could not fit the light curve properly, derived the incorrect values of $\mu \sim 0.3$ mas yr $^{-1}$ and $(V-I)_{S, 0}=0.49$. Second, the color of $(V-I)_{S, 0}=0.58 \pm 0.07$ derived in our analysis is between their spectroscopic and microlensing values, and we found a similar color with their microlensing value when we used RCGs in slightly wider areas around the target, where the RCG distribution gets spread wider along the extinction vector on the CMD. These indicate that their spectroscopic color $(V-I)_{S, 0}=0.71_{-0.02}^{+0.03}$ is correct as well as their and our photometric colors $(V-I)_{S, 0}=0.49$ and $0.58 \pm 0.07$, respectively, which are based on the average color of RCG in wider area, are biased because of low spatial resolution relative to the actual spatial variation of the reddening. Therefore, we conclude that the color difference may be due to the local spatial variation of the extinction in this field rather than the foreground disk scenario. Third, the constraint from $\mathrm{RV}_{\text {helio }}$ is not strong because it is also sufficiently explained by the bulge velocity distribution, which has a large dispersion of $\sigma \sim 100 \mathrm{~km} \mathrm{~s}^{-1}$ (Howard et al. 2008).

Finally, we adopt $90 \%$ of the RCG extinction as the source extinction, i.e., $\left(E(V-I), A_{I}\right)_{S}=0.9 \times\left(E(V-I), A_{I}\right)_{\mathrm{RCG}}=$ $(1.190 \pm 0.064,1.514 \pm 0.044)$ and thus the intrinsic primary source color and magnitude are $(V-I, I)_{S, 0}=(0.714 \pm$ $0.071,18.109 \pm 0.049)$. This is consistent with the spectroscopic source color $(V-I)_{S, 0}=0.71_{-0.02}^{+0.03}$. Note that even if we assumed that the source suffered from the same extinction as that for average RCG, the estimated source angular radius $\theta_{*}$ is consistent with that of $90 \%$ of the average RCG extinction. The source properties are summarized in Table 4 .
Table 4

Source Properties

\begin{tabular}{lccc}
\hline \hline & $V-I(\mathrm{mag})$ & $I(\mathrm{mag})$ & $\theta_{*}(\mu \mathrm{as})$ \\
\hline Apparent & $1.904 \pm 0.009$ & $19.618 \pm 0.006$ & $\cdots$ \\
Intrinsic & $0.714 \pm 0.071$ & $18.104 \pm 0.049$ & $0.757 \pm 0.054$ \\
\hline
\end{tabular}

From Bensby et al. (2017)

\begin{tabular}{lcc}
\hline Effective Temperature & $T_{\text {eff }}{ }^{\mathrm{a}}$ & $5785 \pm 77(\mathrm{~K})$ \\
& $T_{\text {eff }} \mathrm{b}$ & $6616(\mathrm{~K})$ \\
Source Color & $(V-I)_{S, 0}{ }^{\mathrm{a}}$ & $0.71_{-0.02}^{+0.03}(\mathrm{mag})$ \\
& $(V-I)_{S, 0}{ }^{\mathrm{b}}$ & $0.49(\mathrm{mag})$ \\
Absolute Magnitude & $M_{V}{ }^{\mathrm{a}}$ & $3.69(\mathrm{mag})$ \\
Heliocentric RV & $\mathrm{RV}_{\text {helio }}$ & $-46.8\left(\mathrm{~km} \mathrm{~s}^{-1}\right)$ \\
\hline
\end{tabular}

Notes. Bensby et al. (2017) modeled OGLE-2013-BLG-0911 as a 1L1S event.

a Derived from spectroscopy.

${ }^{\mathrm{b}}$ Derived from their microlensing model.

\subsection{Angular Source and Einstein Radius}

With the extinction-free color and magnitude of the source, we can estimate $\theta_{*}$ from a precise empirical $(V-I)$ and $I$ relation

$$
\log _{10}\left(\frac{2 \theta_{*}}{\text { mas }}\right)=0.5014+0.4197(V-I)_{S, 0}-0.2 I_{S, 0},
$$

which is the optimized relation for the color ranges of microlensing observation, derived from the extended analysis of Boyajian et al. (2014). Using Equation (8), we estimated $\theta_{*}=0.757 \pm 0.054 \mu$ as for the best-fit model. We used Equation (8) and took account of the source extinction and its uncertainty into our MCMC calculations to derive the angular Einstein radius $\theta_{\mathrm{E}}$ and the geocentric lens-source 


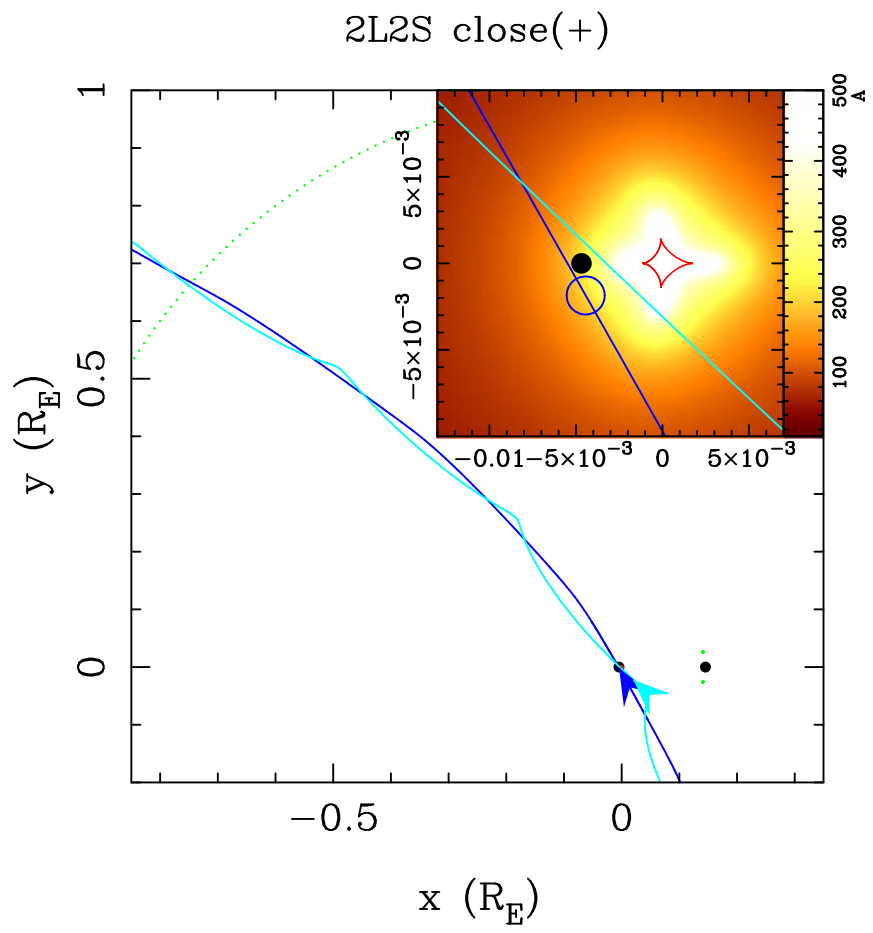

Figure 6. Caustic geometry for the best-fit $2 \mathrm{~L} 2 \mathrm{~S}\left(s<1, u_{0}>0\right)$ model is shown as the red curves, respectively. The blue and light blue curves show the primary and secondary source trajectories with respect to the lens systems, with the arrows indicating the directions of each source motion. The black dots are lens components, and the green dots represent critical curves. The inset shows a zoom-in view around the central caustic. The magnification patterns are described as color maps. The brighter tone denotes higher magnification. The blue circle on the lines indicates the primary source size and its positions is at $t_{0}$.

relative proper motion $\mu_{\mathrm{rel}, \mathrm{G}}$ for each model. The results are summarized in Table 5.

\section{Lens System Properties}

The measurements of both $\theta_{\mathrm{E}}$ and $\pi_{\mathrm{E}}$ enable us to determine the lens mass $M_{L}$ and distance $D_{L}$ directly (Gould 2000; Bennett 2008) as follows:

$$
M_{L}=\frac{c^{2}}{4 G} \theta_{\mathrm{E}}^{2} \frac{D_{S} D_{L}}{D_{S}-D_{L}}=\frac{c^{2}}{4 G} \frac{\mathrm{au}}{\pi_{\mathrm{E}}^{2}} \frac{D_{S}-D_{L}}{D_{S} D_{L}}=\frac{\theta_{\mathrm{E}}}{\kappa \pi_{\mathrm{E}}},
$$

where $D_{L}$ is the lens distances. We derived the probability distributions of the physical parameters of the source and lens systems by calculating their values in each MCMC link. Here, we assumed the primary source mass $M_{S}=1 M_{\odot}$ and the source distance $D_{S}=8 \mathrm{kpc}$. As referred to in Appendix B, we confirmed that the assumptions hardly affect the MCMC posterior distributions for the lens physical parameters except for the lens distance $D_{L}$. We combined the posterior probability distributions of each model weighting by $e^{-\Delta \chi^{2} / 2}$. Figure 8 shows the probability distributions of the lens mass $M_{L}$ and distance $D_{L}$ for the close and wide models, and the final result of the physical parameters are summarized in Table 5. The result indicates that the lens system is an $\mathrm{M}$ dwarf orbited by a massive Jupiter companion at very close $\left(M_{\text {host }}=0.30_{-0.06}^{+0.08} M_{\odot}\right.$, $M_{\text {comp }}=10.1_{-2.2}^{+2.9} M_{\text {Jup }}, a_{\text {exp }}=0.40_{-0.04}^{+0.05}$ au $)$ or wide $\left(M_{\text {host }}=\right.$ $0.28_{-0.08}^{+0.10} M_{\odot}, M_{\text {comp }}=9.9_{-3.5}^{+3.8} M_{\text {Jup }}, a_{\text {exp }}=18.0_{-3.2}^{+3.2}$ au) separation.

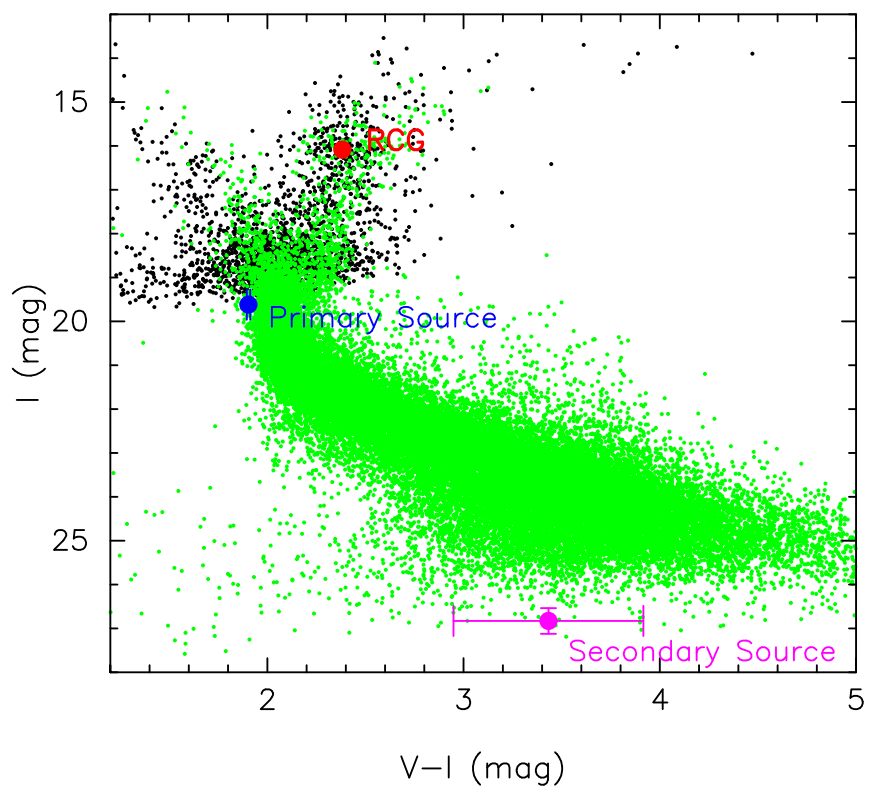

Figure 7. The $(V-I, I) \mathrm{CMD}$ in the standard Kron-Cousins $I$ and Johnson $V$ photometric system. The positions of the primary and secondary source and the centroid of RCG are shown as the blue, magenta, and red circles. The black dots indicate the OGLE-III catalog stars within $1^{\prime}$ of the source. The green dots indicate the Hubble Space Telescope (HST) CMD in Baade's window (Holtzman et al. 1998), whose color and magnitude are matched by using the RCG position.

Table 5

Physical Parameters

\begin{tabular}{lccc}
\hline \hline \multicolumn{1}{c}{ Parameters } & Units & Close & Wide \\
\hline Lens Host Mass, $M_{\text {host }}$ & $M_{\odot}$ & $0.29_{-0.05}^{+0.07}$ & $0.28_{-0.08}^{+0.10}$ \\
Lens Companion Mass, $M_{\text {comp }}$ & $M_{\mathrm{Jup}}$ & $9.51_{-1.69}^{+2.72}$ & $9.92_{-3.45}^{+3.78}$ \\
Lens Distance, $D_{L}$ & $\mathrm{kpc}$ & $3.22_{-0.35}^{+0.47}$ & $3.15_{-0.42}^{+0.53}$ \\
Expected Semimajor Axis, $a_{\mathrm{exp}}{ }^{\mathrm{a}}$ & $\mathrm{au}$ & $0.39_{-0.03}^{+0.05}$ & $17.98_{-3.24}^{+3.21}$ \\
Source Companion Mass, $M_{C}$ & $M_{\odot}$ & $0.137_{-0.016}^{+0.018}$ & $0.137_{-0.014}^{+0.017}$ \\
Distance between Sources, $a_{\mathrm{SC}}$ & $\mathrm{au}$ & $0.225_{-0.004}^{+0.004}$ & $0.225_{-0.003}^{+0.003}$ \\
Angular Einstein Radius, $\theta_{\mathrm{E}}$ & $\mathrm{mas}$ & $0.67_{-0.08}^{+0.10}$ & $0.68_{-0.17}^{+0.14}$ \\
Geocentric Lens-Source Proper & mas yr & $2.54_{-0.30}^{+0.37}$ & $2.50_{-0.65}^{+0.56}$ \\
$\quad$ Motion, $\mu_{\text {rel,G }}$ & & & $26.42_{-1.13}^{+1.15}$ \\
Predicted Lens Magnitude, $V_{L}$ & mag & $22.80_{-0.83}^{+0.88}$ \\
Predicted Lens Magnitude, $I_{L}$ & $\mathrm{mag}$ & $19.99_{-0.78}^{+0.79}$ \\
Predicted Lens Magnitude, $H_{L}$ & $\mathrm{mag}$ & $19.64_{-0.76}^{+0.78}$ \\
Predicted Lens Magnitude, $K_{L}$ & $\mathrm{mag}$ & & \\
\hline
\end{tabular}

Notes. The median value and $68.3 \%$ confidence interval derived from MCMC. Here, we assume $D_{S}=8 \mathrm{kpc}$ and $M_{S}=1 M_{\odot}$ except for the lens magnitudes. ${ }^{\mathrm{a}} a_{\exp }=\sqrt{3 / 2} a_{\perp}$.

We evaluated the expected apparent magnitude of the lens brightness by conducting a Bayesian analysis based on the observed $t_{\mathrm{E}}, \theta_{\mathrm{E}}$, and $\pi_{\mathrm{E}}$ and prior probabilities from a standard Galactic model (Sumi et al. 2011). Here, we evaluated the extinction in front of the lens given by

$$
A_{i, L}=\frac{1-e^{-D_{L} / h_{\mathrm{dust}}}}{1-e^{-D_{S} / h_{\mathrm{dust}}}} A_{i, S},
$$

where the index $i$ corresponds to the passband $V, I, H$, and $K$, and the $h_{\text {dust }}=(0.1 \mathrm{kpc}) / \sin |b|$ is a scale length of the dust toward the event (Bennett et al. 2015). The lens brightness and 
Close Model

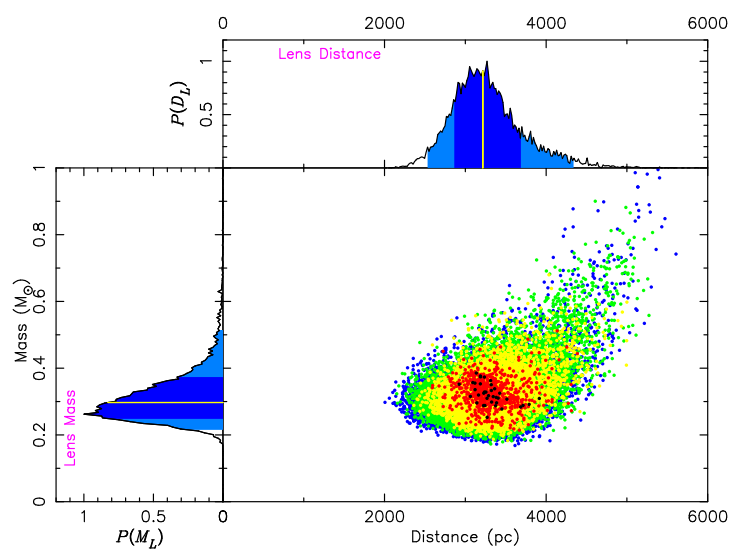

Wide Model

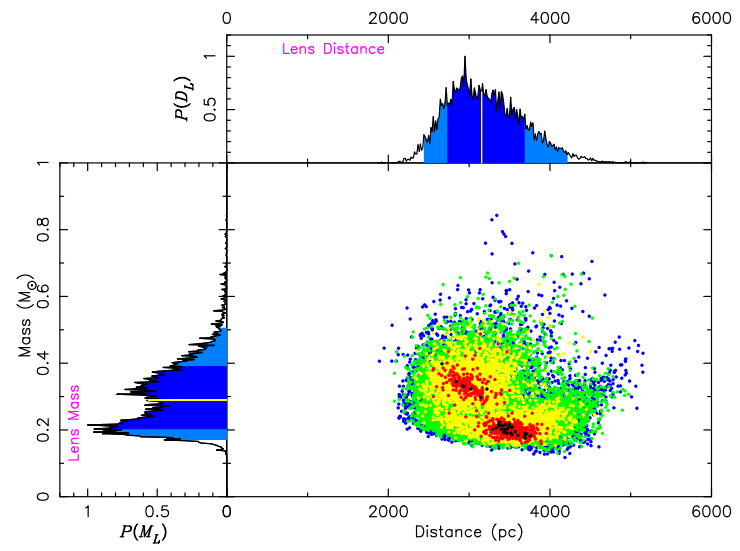

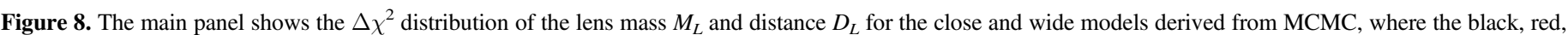

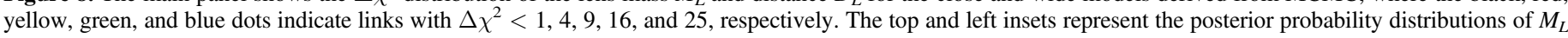

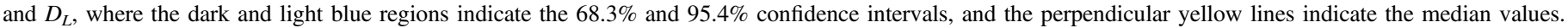

the extinction values are estimated from the color-color and mass-luminosity relations of main-sequence stars (Henry \& McCarthy 1993; Kenyon \& Hartmann 1995; Kroupa \& Tout 1997) and the extinction law in Nishiyama et al. (2009), respectively. We also estimated the source magnitudes in $\mathrm{H}$ and $K$-bands from Kenyon \& Hartmann (1995), taking into account a $10 \%$ uncertainty. Figure 9 represents the apparent lens magnitudes in each band derived from the Bayesian analysis. The dark and light blue regions indicate the $68.3 \%$ and $95.4 \%$ confidence intervals, and the vertical blue lines indicate the median values. The vertical solid and dashed red lines are the source magnitudes and their $1 \sigma$ uncertainties in each passband. The relationship between the heliocentric and geocentric relative proper motion is

$$
\boldsymbol{\mu}_{\mathrm{rel}, \mathrm{H}}=\boldsymbol{\mu}_{\mathrm{rel}, \mathrm{G}}+\frac{\pi_{\mathrm{rel}}}{\mathrm{au}} \boldsymbol{v}_{\oplus}
$$

where $\pi_{\text {rel }}=\operatorname{au}\left(D_{L}^{-1}-D_{S}^{-1}\right)$ and $\boldsymbol{v}_{\oplus}=\left(v_{\oplus, N}, v_{\oplus, E}\right)=(-2.91$, 9.44) $\mathrm{km} \mathrm{s}^{-1}$ are the relative lens-source parallax and the instant velocity of Earth on the plane of the sky at the reference time, respectively. The heliocentric relative proper motion is

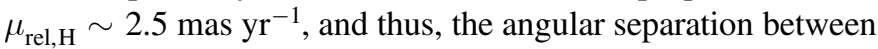
the source and lens would be $\sim 15$ mas in 2019. Bhattacharya et al. (2017) have demonstrated the feasibility of HST followup observations to measure the separation between the source and the lens with 12 mas when the lens is not too much fainter than the source. (The current state of technical arts for high angular resolution analysis is detailed in Bhattacharya et al. 2018.) Hence, it might benefit from a high-resolution follow-up observation in order to constrain the physical parameters of the lens system. However, we note that the four degenerate solutions have parallax vectors $\pi_{\mathrm{E}}$ with amplitudes, directions, and uncertainties approximately similar to each other, and thus, it is unlikely that the degenerate solutions are resolved by highresolution follow-up observations.

\section{Summary and Discussion}

We have presented our analysis of the microlensing event OGLE-2013-BLG-0911. Previous research on the event
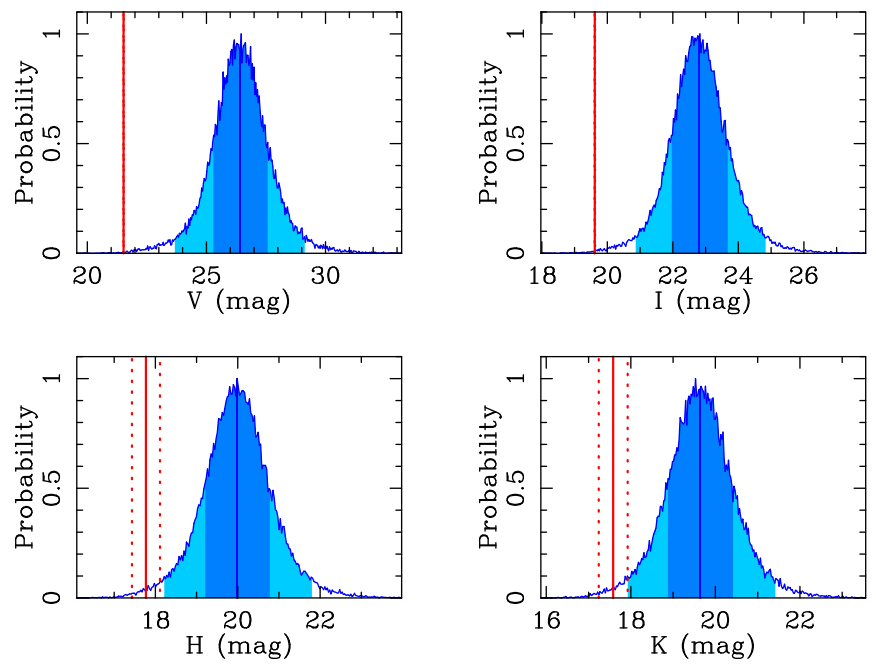

Figure 9. Posterior probabilities of the lens apparent magnitudes derived from the Bayesian analysis with the observed $t_{\mathrm{E}}, \theta_{\mathrm{E}}$, and $\pi_{\mathrm{E}}$ and prior probabilities from a standard Galactic model. The dark and light blue regions indicate the $68.3 \%$ and $95.4 \%$ confidence intervals, and the perpendicular blue lines indicate the median values. The vertical solid and dashed red lines are the source magnitudes and their $1 \sigma$ uncertainties in each passband.

(Shvartzvald et al. 2016) reported that the lensing anomaly could be explained by a planetary mass ratio, $q \approx 3 \times 10^{-4}$. From a detailed grid search analysis, however, we found that a binary mass ratio $q \approx 3 \times 10^{-2}$ is preferred over a planetary mass ratio to explain the light curve. Finally, we conclude that the lens system consists of an $\mathrm{M}$ dwarf orbited by a massive Jupiter companion at very close $\left(M_{\text {host }}=0.30_{-0.06}^{+0.08} M_{\odot}\right.$, $\left.M_{\text {comp }}=10.1_{-2.2}^{+2.9} M_{\mathrm{Jup}}, a_{\mathrm{exp}}=0.40_{-0.04}^{+0.05} \mathrm{au}\right)$ or wide $\left(M_{\mathrm{host}}=\right.$ $0.28_{-0.08}^{+0.10} M_{\odot}, M_{\text {comp }}=9.9_{-3.5}^{+3.8} M_{\text {Jup }}, a_{\text {exp }}=18.0_{-3.2}^{+3.2}$ au $)$ separation.

Microlensing light curves generally provide much more precise estimation of the mass ratio than that of the absolute lens mass. Bond et al. (2004) defined the mass ratio boundary between BDs and planets as $q=0.03$ in order to distinguish between planetary and stellar binary (including BD) microlensing events. For this event, the best-fit mass ratio is slightly above the mass ratio boundary of $q=0.03$. On the other hand, the median mass of the companion is slightly below the lower limit of the BD mass of $13 M_{\text {Jup }}$. Therefore, it is ambiguous to 
classify the companion as a BD or a planet. In fact, these boundaries are somewhat arbitrary, and it might be nonsense to classify such an ambiguous companion according to the boundaries. However, the formation mechanisms for BDs and planets are likely to be different, and the object near the boundaries could have been formed by either formation mechanism. Therefore, it would be very important to probe the distribution of intermediate-mass companions of $\sim 13 M_{\text {Jup }}$.

Missing the best lens model explanation to the observed microlensing light-curve data might have serious impacts on any statistical microlensing analysis incorporating those modeling results. For instance, Shvartzvald et al. (2016) suggests that there is a possible $\mathrm{BD}$ deficit corresponding to $q \sim 10^{-2}$ in their detection-efficiency-corrected mass ratio function. However, we found OGLE-2013-BLG-0911, which was adopted as a planetary sample in their analysis, would correspond to the position of the BD deficit, which would affect their result to some extent. The reason why they missed the best solution would be the very small/wide projected separation $s \approx 0.2$ or $\approx 7$. They explored the $s$ parameter space of $0.3<s<3$ in their grid search analysis. It is known that a central caustic size is approximately proportional to not only $q$ but also $s^{2}$ (for $s \ll 1$ ) and $s^{-2}$ (for $s \gg 1$ ) (Chung et al. 2005). Therefore, when we model microlensing light curves with perturbations caused by possibly small-size central caustics, we should suspect the possibilities of not only very low-mass but also very close and wide-lens companions. The detection efficiency for companions with such extremely close and wide separation is much lower than that with $s \approx 1$ (Suzuki et al. 2016). Hence, even a small number of detections may be important in the statistical analysis.

The successful discovery of the best-fit model depends on the initial parameters for the MCMC fitting. Currently, the initial parameters for modeling binary-lens events are mainly based on the experiences of the modelers or the brute-force of the grid search analysis across a wide range of parameter spaces. The systematic analysis of many events relies on the latter method. However, it would not work if the best-fit solutions are out of range of the grid search, which happened on this event OGLE-2013-BLG-0911. Broadening the search range as possible is a straightforward way to avoid the problem. However, it is computationally expensive and getting more difficult for statistical analysis, including hundreds of stellar binary events in the recent high-cadence surveys by MOA, OGLE, and KMTNet (Kim et al. 2016). Furthermore, the Wide Field Infrared Survey Telescope (Spergel et al. 2015) will be launched in 2025 and is expected to discover $\sim 54,000$ microlensing events $\left(\left|u_{0}\right|<3\right)$ with thousands of binary-lens events, including $\sim 1400$ bound exoplanets with masses of $0.1<M_{p} / M_{\oplus}<10^{4}$ (Penny et al. 2019). We should consider a new method to efficiently search for the best binary-lens solutions. Bennett et al. (2012) applied a different parameterization for wide-separate binary events. Khakpash et al. (2019) proposed an algorithm that can rapidly evaluate many binarylens light curves and estimate the physical parameters of the lens systems, which is successful for very low-mass-ratio events but less so for higher mass-ratio events.

There are only four discoveries of $\mathrm{BD}$ companions to $\mathrm{M}$ dwarfs within 10 pc from solar system (Winters et al. 2018), while approximately $200 \mathrm{M}$ dwarfs are known to exist within 10 pc (Henry et al. 2006, 2016) and much effort has been dedicated to detect such BD companions (Henry \& McCarthy 1990;
Dieterich et al. 2012). Because of their scarcity, incoming new BD discoveries around $\mathrm{M}$ dwarfs provide valuable constraints on the formation and evolution theories of stars, BDs, and planets. Microlensing is a powerful method to probe the $\mathrm{BD} /$ massive-planet occurrence frequency across orbital radii $0.1 \leqslant a \leqslant 10$ au around low-mass hosts such as $\mathrm{M}$ dwarfs and even BDs (Gaudi 2002), which is challenging for other exoplanet detection methods. Although microlensing samples generally cannot provide some information such as host metallicity and eccentricity, microlensing can provide both their masses and orbital separations. It is very important to uncover the distributions of BD properties by microlensing.

We would like to thank the anonymous referee who helped to greatly improve our paper. The OGLE project has received funding from the National Science Centre, Poland, grant MAESTRO 2014/14/A/ST9/00121 to A.U. CITEUC is funded by National Funds through FCT-Foundation for Science and Technology (project: UID/Multi/00611/2013) and FEDER - European Regional Development Fund through COMPETE 2020-Operational Programme Competitiveness and Internationalization (project: POCI-01-0145-FEDER006922). D.P.B., A.B., and D.S. were supported by NASA through grant NASA-NNX12AF54G. Work by C.R. was supported by an appointment to the NASA Postdoctoral Program at the Goddard Space Flight Center, administered by USRA through a contract with NASA. Work by N.K. is supported by JSPS KAKENHI grant No. JP15J01676. Work by Y.H. is supported by JSPS KAKENHI grant No. JP1702146. N.J.R. is a Royal Society of New Zealand Rutherford Discovery Fellow. This work was supported by JSPS KAKENHI grant No. JP17H02871. This research was supported by the I-CORE program of the Planning and Budgeting Committee and the Israel Science Foundation, grant $1829 / 12$. D.M. acknowledges support by the U.S.-Israel Binational Science Foundation. Work by C.H. was supported by the grant (2017R1A4A1015178) of National Research Foundation of Korea. Work by W.Z., Y.K.J., and A.G. was supported by AST1516842 from the US NSF. W.Z., I.G.S., and A.G. were supported by JPL grant 1500811. Y.T. acknowledges the support of DFG priority program SPP 1992 "Exploring the Diversity of Extrasolar Planets" (WA 1047/ 11-1). K.H. acknowledges support from STFC grant ST/ $\mathrm{R} 000824 / 1$.

\section{Appendix A Calibration for the Source Magnitude}

We derived the apparent magnitude and color of the source from the measurements of CT13-I and $V$ that were made during the time of high magnification. We basically followed the procedure described in Bond et al. (2017) in order to convert the CT13 instrumental magnitudes into the standard ones. We cross-referenced isolated stars around $2^{\prime}$ of the source between the CT13 catalog reduced by DoPHOT (Schechter et al. 1993) and the OGLE-III catalog (Szymański et al. 2011). We found the following relation:

$$
\begin{aligned}
I_{\mathrm{O} 3}-I_{\mathrm{CT} 13} & =(27.070 \pm 0.011)-(0.032 \pm 0.006)(V-I)_{\mathrm{CT} 13} \\
V_{\mathrm{O} 3}-V_{\mathrm{CT} 13} & =(27.851 \pm 0.017)-(0.101 \pm 0.011)(V-I)_{\mathrm{CT} 13} .
\end{aligned}
$$

Consequently, we obtained the apparent color and magnitude of the source, $(V-I, I)_{S, \mathrm{CT} 13}=(1.904 \pm 0.009,19.618 \pm 0.006)$. Moreover, we also derived the source color and magnitude from 

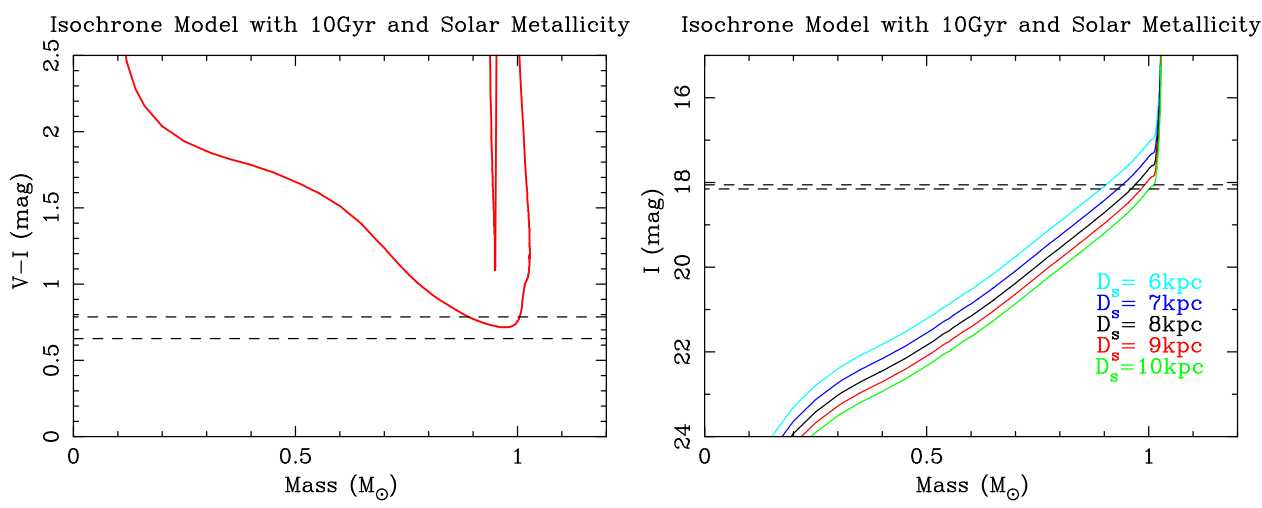

Figure 10. PARSEC stellar isochrone model with solar metallicity and $10 \mathrm{Gyr}$ age. The areas enclosed by the horizontal dashed lines represent the $1 \sigma$ ranges for the observed intrinsic source color $(V-I)_{S, 0}=0.714 \pm 0.071$ (right panel) and magnitude $I_{S, 0}=18.104 \pm 0.049$ (left panel), respectively.

the measurements of OGLE- $I$ and $V$ for confirmation. We used Equation (1) in Udalski et al. (2015) to calibrate the OGLE-IV instrumental magnitudes into the standard ones. We applied $\Delta Z P_{I}=-0.056, \quad \Delta Z P_{V}=0.133, \quad \epsilon_{I}=-0.005 \pm$ 0.003 , and $\epsilon_{V}=-0.077 \pm 0.001$ for Equation (1) in Udalski et al. (2015), which were obtained by private communication with the OGLE collaboration. Finally, we derived the apparent source color and magnitude from OGLE-I and $V,(V-I, I)_{S, \mathrm{O} 4}=(1.880 \pm 0.009,19.594 \pm 0.006)$.

\section{Appendix B \\ Impact of the Assumption for $M_{S}$ and $D_{S}$}

We tested how the assumption of the fixed $M_{S}=1 M_{\odot}$ and $D_{S}=8 \mathrm{kpc}$ impacted the final results. Figure 10 represents the PARSEC stellar isochrone with solar metallicity and $10 \mathrm{Gyr}$ age. Comparing the isochrone to the observed intrinsic source color and magnitude $(V-I, I)_{S, 0}=(0.714 \pm 0.071,18.104 \pm$ 0.049 ), we can state that the source mass and distance are likely to be in the ranges of $0.9 \leqslant M_{S} / M_{\odot} \leqslant 1.0$ and $6 \mathrm{kpc} \leqslant D_{S} \leqslant 10 \mathrm{kpc}$, respectively. In these likely ranges, we conducted light-curve modeling for $1 \mathrm{~L} 2 \mathrm{~S}, 2 \mathrm{~L} 1 \mathrm{~S}$, and $2 \mathrm{~L} 2 \mathrm{~S}$ with all 15 combinations of the fixed $M_{S}=(0.9,0.95,1.0) M_{\odot}$ and $D_{S}=(6,7,8,9,10) \mathrm{kpc}$. We found that the fixed values have little effect on the best-fit $\chi^{2}$ value, and the MCMC posterior distributions for the lens physical parameters are consistent with each other within $1 \sigma$ except for the lens distance $D_{L}$. Therefore, we conclude that the assumptions for $M_{S}$ and $D_{S}$ do not significantly affect the final results except $D_{L}$.

\section{ORCID iDs}

Shota Miyazaki iㅏ https://orcid.org/0000-0001-9818-1513 David P. Bennett ㄴ) https://orcid.org/0000-0001-8043-8413 Yossi Shvartzvald (i) https://orcid.org/0000-0003-1525-5041 Valerio Bozza (1) https://orcid.org/0000-0003-4590-0136 Jennifer C. Yee (1) https://orcid.org/0000-0001-9481-7123 Nicholas Rattenbury (1) https://orcid.org/0000-00015069-319X

Naoki Koshimoto (1) https://orcid.org/0000-0003-2302-9562

Akihiko Fukui $\odot$ https://orcid.org/0000-0002-4909-5763

Y. Itow (1) https://orcid.org/0000-0002-8198-1968

I. Kondo (i) https://orcid.org/0000-0002-3401-1029

J. Skowron 1 () https://orcid.org/0000-0002-2335-1730

P. Pietrukowicz (1) https://orcid.org/0000-0002-2339-5899

K. Ulaczyk (1) https://orcid.org/0000-0001-6364-408X
M. Albrow (i) https://orcid.org/0000-0003-3316-4012

A. Gal-Yam (1) https://orcid.org/0000-0002-3653-5598

R. W. Pogge (ㄷ) https://orcid.org/0000-0003-1435-3053

Y. Tsapras (1) https://orcid.org/0000-0001-8411-351X

E. Bachelet (1) https://orcid.org/0000-0002-6578-5078

K. Horne (i) https://orcid.org/0000-0003-1728-0304

C. Snodgrass (i) https://orcid.org/0000-0001-9328-2905

J. Wambsganss (1) https://orcid.org/0000-0002-8365-7619

I. A. Steele (1) https://orcid.org/0000-0001-8397-5759

G. D'Ago (1) https://orcid.org/0000-0001-9697-7331

T. C. Hinse (1) https://orcid.org/0000-0001-8870-3146

N. Kains (i) https://orcid.org/0000-0001-8803-6769

L. Mancini (i) https://orcid.org/0000-0002-9428-8732

M. Rabus 스 https://orcid.org/0000-0003-2935-7196

S. Rahvar (ib https://orcid.org/0000-0002-7084-5725

N. Peixinho 난 https://orcid.org/0000-0002-6830-476X

\section{References}

Alard, C. 2000, A\&AS, 144, 363

Alard, C., \& Lupton, R. H. 1998, ApJ, 503, 325

Albrow, M. D., Horne, K., Bramich, D. M., et al. 2009, MNRAS, 397, 2099

André, P., Ward-Thompson, D., \& Greaves, J. 2012, Sci, 337, 69

Armitage, P. J., \& Bonnell, I. A. 2002, MNRAS, 330, L11

Batista, V., Dong, S., Gould, A., et al. 2009, A\&A, 508, 467

Bennett, D. P. 2008, in Exoplanets, ed. J. W. Mason (Berlin: Springer), 47

Bennett, D. P. 2010, ApJ, 716, 1408

Bennett, D. P., Batista, V., Bond, I. A., et al. 2014, ApJ, 785, 155

Bennett, D. P., Bhattacharya, A., Anderson, J., et al. 2015, ApJ, 808, 169

Bennett, D. P., Bond, I. A., Udalski, A., et al. 2008, ApJ, 684, 663

Bennett, D. P., \& Rhie, S. H. 1996, ApJ, 472, 660

Bennett, D. P., Sumi, T., Bond, I. A., et al. 2012, ApJ, 757, 119

Bennett, D. P., Udalski, A., Han, C., et al. 2018, AJ, 155, 141

Bensby, T., Adén, D., Meléndez, J., et al. 2011, A\&A, 533, A134

Bensby, T., Feltzing, S., Gould, A., et al. 2017, A\&A, 605, A89

Bensby, T., Yee, J. C., Feltzing, S., et al. 2013, A\&A, 549, A147

Bhattacharya, A., Beaulieu, J.-P., Bennett, D. P., et al. 2018, AJ, 156, 289

Bhattacharya, A., Bennett, D. P., Anderson, J., et al. 2017, AJ, 154, 59

Bond, I. A., Abe, F., Dodd, R. J., et al. 2001, MNRAS, 327, 868

Bond, I. A., Bennett, D. P., Sumi, T., et al. 2017, MNRAS, 469, 2434

Bond, I. A., Udalski, A., Jaroszyński, M., et al. 2004, ApJL, 606, L155

Borucki, W. J., Koch, D., Basri, G., et al. 2010, Sci, 327, 977

Boss, A. P. 1997, Sci, 276, 1836

Boss, A. P. 2001, ApJL, 551, L167

Boyajian, T. S., van Belle, G., \& von Braun, K. 2014, AJ, 147, 47

Bramich, D. M. 2008, MNRAS, 386, L77

Bramich, D. M., Horne, K., Albrow, M. D., et al. 2013, MNRAS, 428, 2275

Bressan, A., Marigo, P., Girardi, L., et al. 2012, MNRAS, 427, 127

Burrows, A., Hubbard, W. B., Saumon, D., \& Lunine, J. I. 1993, ApJ, 406, 158

Carnero Rosell, A., Santiago, B., \& dal Ponte, M. 2019, MNRAS, 489, 5301

Choi, J.-Y., Shin, I.-G., Han, C., et al. 2012, ApJ, 756, 48

Chung, S.-J., Han, C., Park, B.-G., et al. 2005, ApJ, 630, 535

Claret, A., \& Bloemen, S. 2011, A\&A, 529, A75 
Dieterich, S. B., Henry, T. J., Golimowski, D. A., Krist, J. E., \& Tanner, A. M. 2012, AJ, 144, 64

Dominik, M. 1999, A\&A, 349, 108

Dominik, M., Bachelet, E., Bozza, V., et al. 2019, MNRAS, 484, 5608

Dominik, M., Jørgensen, U. G., Rattenbury, N. J., et al. 2010, AN, 331, 671

Duchêne, G., \& Kraus, A. 2013, ARA\&A, 51, 269

Gaudi, B. S. 1998, ApJ, 506, 533

Gaudi, B. S. 2002, arXiv:astro-ph/0206494

Gaudi, B. S., Beaulieu, J. P., Bennett, D. P., et al. 2009, astro2010: The Astronomy and Astrophysics Decadal Survey, Science White Papers, 85

González Hernández, J. I., \& Bonifacio, P. 2009, A\&A, 497, 497

Gorbikov, E., Brosch, N., \& Afonso, C. 2010, Ap\&SS, 326, 203

Gould, A. 1992, ApJ, 392, 442

Gould, A. 2000, ApJ, 542, 785

Gould, A. 2004, ApJ, 606, 319

Gould, A., Dong, S., Bennett, D. P., et al. 2010, ApJ, 710, 1800

Gould, A., Udalski, A., An, D., et al. 2006, ApJL, 644, L37

Gould, A., \& Welch, D. L. 1996, ApJ, 464, 212

Grether, D., \& Lineweaver, C. H. 2006, ApJ, 640, 1051

Griest, K., \& Hu, W. 1992, ApJ, 397, 362

Griest, K., \& Safizadeh, N. 1998, ApJ, 500, 37

Han, C., \& Gould, A. 1997, ApJ, 480, 196

Han, C., Udalski, A., Sumi, T., et al. 2017, ApJ, 843, 59

Hayashi, C. 1981, in Fundamental Problems in the Theory of Stellar Evolution, ed. D. Sugimoto, D. Q. Lamb, \& D. N. Schramm (Dordrecht: D. Reidel), 113

Henry, T. J., Jao, W.-C., Subasavage, J. P., et al. 2006, AJ, 132, 2360

Henry, T. J., Jao, W.-C., Winters, J. G., et al. 2016, AAS Meeting Abstracts, $227,142.01$

Henry, T. J., \& McCarthy, D. W., Jr. 1990, ApJ, 350, 334

Henry, T. J., \& McCarthy, D. W., Jr. 1993, AJ, 106, 773

Holtzman, J. A., Watson, A. M., Baum, W. A., et al. 1998, AJ, 115, 1946

Howard, C. D., Rich, R. M., Reitzel, D. B., et al. 2008, ApJ, 688, 1060

Ida, S., \& Lin, D. N. C. 2005, ApJ, 626, 1045

Johnson, J. A., Howard, A. W., Marcy, G. W., et al. 2010, PASP, 122, 149

Jung, Y. K., Udalski, A., Bond, I. A., et al. 2017a, ApJ, 841, 75

Jung, Y. K., Udalski, A., Yee, J. C., et al. 2017b, AJ, 153, 129

Kenyon, S. J., \& Hartmann, L. 1995, ApJS, 101, 117

Khakpash, S., Penny, M., \& Pepper, J. 2019, AJ, 158, 9

Kim, S.-L., Lee, C.-U., Park, B.-G., et al. 2016, JKAS, 49, 37

Kroupa, P., \& Tout, C. A. 1997, MNRAS, 287, 402

Kumar, S. S. 1962, Institute for Space Studies Report No. X-644-62-78
Laughlin, G., Bodenheimer, P., \& Adams, F. C. 2004, ApJL, 612, L73

Lissauer, J. J. 1993, ARA\&A, 31, 129

Loeb, A., \& Sasselov, D. 1995, ApJL, 449, L33

Luhman, K. L. 2012, ARA\&A, 50, 65

Mao, S., \& Paczynski, B. 1991, ApJL, 374, L37

Marcy, G. W., \& Butler, R. P. 2000, PASP, 112, 137

Matzner, C. D., \& Levin, Y. 2005, ApJ, 628, 817

Miyazaki, S., Sumi, T., Bennett, D. P., et al. 2018, AJ, 156, 136

Mordasini, C., Alibert, Y., Benz, W., \& Naef, D. 2009, A\&A, 501, 1161

Nakajima, T., Oppenheimer, B. R., Kulkarni, S. R., et al. 1995, Natur, 378, 463

Nataf, D. M., Gould, A., Fouqué, P., et al. 2013, ApJ, 769, 88

Nishiyama, S., Tamura, M., Hatano, H., et al. 2009, ApJ, 696, 1407

Paczynski, B. 1986, ApJ, 304, 1

Penny, M. T., Gaudi, B. S., Kerins, E., et al. 2019, ApJS, 241, 3

Poindexter, S., Afonso, C., Bennett, D. P., et al. 2005, ApJ, 633, 914

Pollack, J. B., Hubickyj, O., Bodenheimer, P., et al. 1996, Icar, 124, 62

Ranc, C., Cassan, A., Albrow, M. D., et al. 2015, A\&A, 580, A125

Rattenbury, N. J., Bond, I. A., Skuljan, J., \& Yock, P. C. M. 2002, MNRAS, 335,159

Ryu, Y.-H., Yee, J. C., Udalski, A., et al. 2018, AJ, 155, 40

Sako, T., Sekiguchi, T., Sasaki, M., et al. 2008, ExA, 22, 51

Schechter, P. L., Mateo, M., \& Saha, A. 1993, PASP, 105, 1342

Shin, I.-G., Yee, J. C., Gould, A., et al. 2019, AJ, 158, 199

Shvartzvald, Y., \& Maoz, D. 2012, MNRAS, 419, 3631

Shvartzvald, Y., Maoz, D., Udalski, A., et al. 2016, MNRAS, 457, 4089

Skowron, J., Udalski, A., Gould, A., et al. 2011, ApJ, 738, 87

Skowron, J., Udalski, A., Kozłowski, S., et al. 2016, AcA, 66, 1

Smith, M. C., Mao, S., \& Paczyński, B. 2003, MNRAS, 339, 925

Spergel, D., Gehrels, N., Baltay, C., et al. 2015, arXiv:1503.03757

Sumi, T., Abe, F., Bond, I. A., et al. 2003, ApJ, 591, 204

Sumi, T., Kamiya, K., Bennett, D. P., et al. 2011, Natur, 473, 349

Suzuki, D., Bennett, D. P., Sumi, T., et al. 2016, ApJ, 833, 145

Szymański, M. K., Udalski, A., Soszyński, I., et al. 2011, AcA, 61, 83

Tanigawa, T., \& Tanaka, H. 2016, ApJ, 823, 48

Tsapras, Y., Street, R., Horne, K., et al. 2009, AN, 330, 4

Udalski, A., Szymanski, M., Stanek, K. Z., et al. 1994, AcA, 44, 165

Udalski, A., Szymański, M. K., \& Szymański, G. 2015, AcA, 65, 1

Verde, L., Peiris, H. V., Spergel, D. N., et al. 2003, ApJS, 148, 195

Winters, J. G., Irwin, J., Newton, E. R., et al. 2018, AJ, 155, 125

Wozniak, P. R. 2000, AcA, 50, 421

Yee, J. C., Shvartzvald, Y., Gal-Yam, A., et al. 2012, ApJ, 755, 102

Yoo, J., DePoy, D. L., Gal-Yam, A., et al. 2004, ApJ, 603, 139 